\title{
Rheological Measurement of Suspensions Without Slippage: Experiment and Model
}

\author{
Alex Olivas \\ Michelle A. Helsel \\ Nicos S. Martys \\ Chiara F. Ferraris \\ William L. George \\ Raissa Ferron
}




\title{
Rheological Measurement of Suspensions Without Slippage: Experiment and Model
}

\author{
Alex Olivas \\ Michelle A. Helsel \\ Nicos S. Martys \\ Chiara F. Ferraris \\ Materials and Construction Research Division \\ Engineering Laboratory \\ William L. George \\ Applied and Computational Mathematics Division \\ Information Technology Laboratory \\ Raissa Ferron \\ The University of Texas at Austin
}

This publication is available free of charge from:

https://doi.org/10.6028/NIST.TN.1946

December 2016

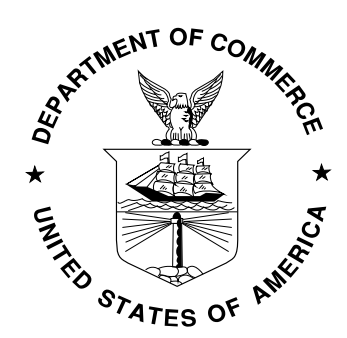

U.S. Department of Commerce
Penny Pritzker, Secretary

National Institute of Standards and Technology Willie May, Under Secretary of Commerce for Standards and Technology and Director 
Certain commercial entities, equipment, or materials may be identified in this document in order to describe an experimental procedure or concept adequately. Such identification is not intended to imply recommendation or endorsement by the National Institute of Standards and Technology, nor is it intended to imply that the entities, materials, or equipment are necessarily the best available for the purpose.

National Institute of Standards and Technology Technical Note 1946 Natl. Inst. Stand. Technol. Tech. Note 1946, 45 pages (December 2016) CODEN: NTNOEF

This publication is available free of charge from: https://doi.org/10.6028/NIST.TN.1946 


\begin{abstract}
Rheological measurements of suspensions are often performed using a rotational rheometer. In this type of rheometer, the tested fluid is sheared between two surfaces, one of which is rotating to generate a laminar flow of the fluid between the surfaces (i.e., a Couette flow). Manufacturers of commercially available rheometers generally recommend the use of a standard oil of known viscosity to verify that the rheometer is operating correctly. However, in the case of concrete rheometers, this approach would require large volumes of oil and was deemed not economically feasible by two international studies [1] [2]. The conclusion of those international studies was that the optimal approach to calibrate concrete rheometers would be to develop a non-Newtonian standard reference material (SRM) that contained inclusions similar in size to aggregates used commonly in concrete. This could be achieved by using a multi-stage approach where each stage corresponds to a different level of complexity of the fluid. The first stage would be to create a paste reference material, as was done in the SRM 2492 [3]. The second stage would be to mimic the mortar phase of a concrete, and SRM 2493 [4], with $1 \mathrm{~mm}$ beads added to SRM 2492, accomplishes that goal. The third, and final stage, is the creation of SRM 2497 for concrete, with larger beads added to SRM 2493, which is currently in development at National Institute of Standards and Technology (NIST).
\end{abstract}

During the certification of SRM 2493, it was found that differences in rheometer geometry affect the accuracy of the rheological measurements. In order to gain fundamental insight about the impact that different rheometer geometries have on measurements of suspensions, a comprehensive analysis was conducted on three different rheometer families. The analysis included both experimental testing and computer simulation. The comparison between the model and rheological results showed that the increased viscosity due to the addition of the $1 \mathrm{~mm}$ beads to SRM 2492 was significantly higher in the Couette model than in the experimental data. It was also determined that some geometries, such as a double spiral, resulted in a higher viscosity than a simple serrated cylinder or vane. This finding led to the inference that slippage should also be considered. Ultimately, this report highlights that industrial rheometers experience slippage issues caused by their choice of geometry and their internal boundary conditions (free surfaces), and discusses the most accurate alternative available for calibrating rheometers. 


\section{Table of Contents}

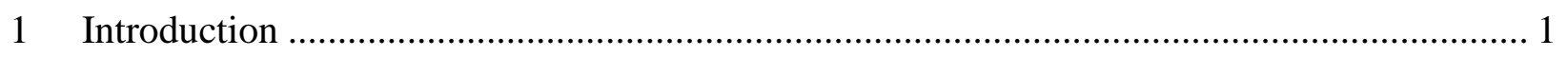

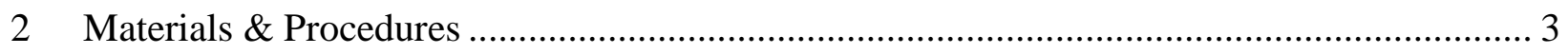

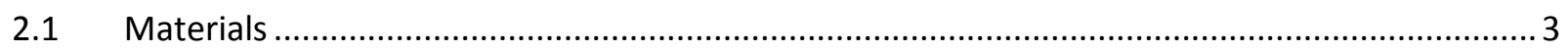

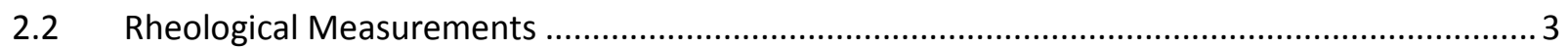

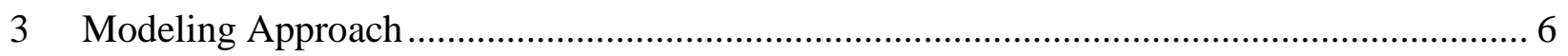

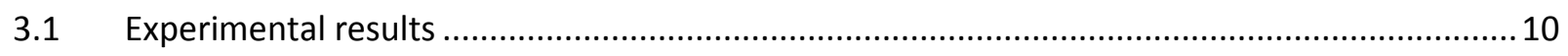

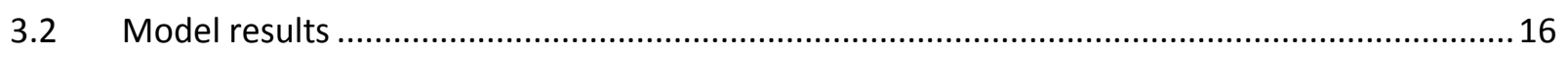

3.3 Confined rheological measurements ............................................................................ 19

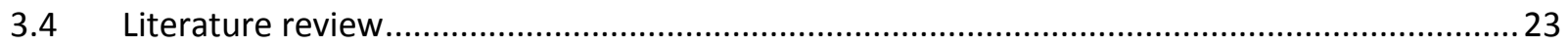

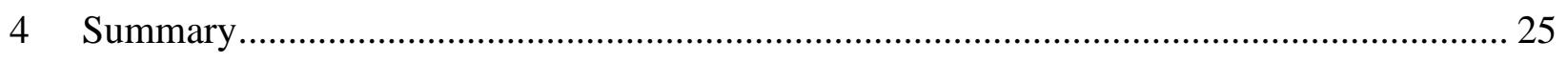

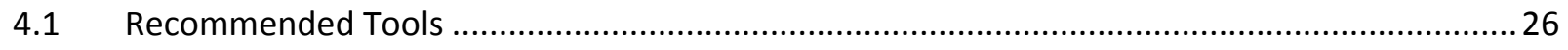

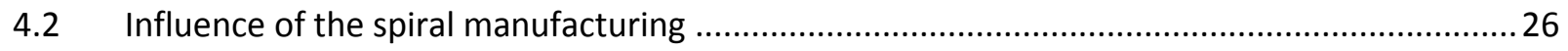

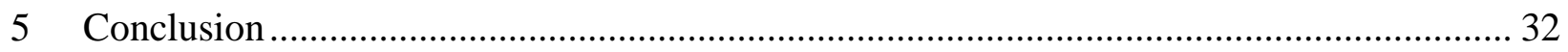

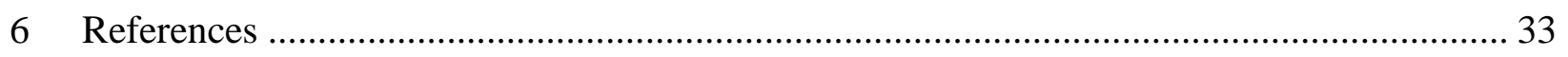

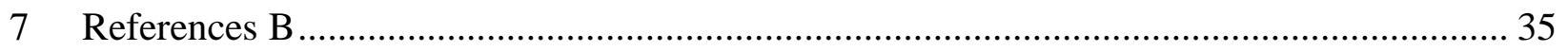




\section{List of Figures}

Figure 1: Three geometry types used for testing, each representing a different spindle family.... 4

Figure 2: Simulation model data and predicted data from scaling parameters are compared to experimental data results. Uncertainty was found to be approximately $5 \%$ for the experimental data [4].

Figure 3: Viscosity curve comparison of the calibrated experimental data when SRM paste ( $0 \%$ beads) was sheared using all three spindles. The flow curves are compared to their reference curve shown in black, which represents the SRM2492 certified values. Uncertainty was found to be approximately $5 \%$ for the experimental data from [4]..... 11

Figure 4: Behavior of SRM paste at low-range shear rates $\left(<0.1 \mathrm{~s}^{-1}\right)$, corresponding to the orange-shaded region in Figure 3. Uncertainty was found to be approximately $5 \%$ for the

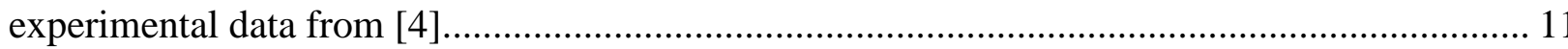

Figure 5: Behavior of SRM paste at mid-range shear rates $\left(0.1 \mathrm{~s}^{-1}<1.0 \mathrm{~s}^{-1}\right)$, corresponding to the blue-shaded area from Figure 3. Uncertainty was found to be approximately $5 \%$ for the experimental data from [4]......

Figure 6: Behavior of SRM paste at high-range shear rates $\left(1.0 \mathrm{~s}^{-1}<50 \mathrm{~s}^{-1}\right)$, corresponding to the red shaded area from Figure 3. The uncertainty was found to be approximately $5 \%$ for the

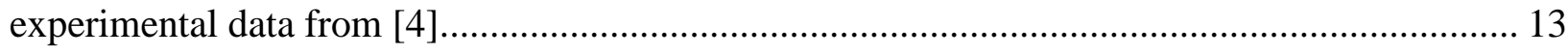

Figure 7: Viscosity curve at concentrations of $20 \%$ and $40 \%$, by volume, of glass beads. The uncertainty was estimated to be $10 \%$ of the viscosity. Full discussion on the uncertainty can be found in the full report $[13,4]$.

Figure 8: One example of relative viscosity $(\mu \mathrm{R})$, for double spiral and a material set of $40 \%$ beads by volume. The $\mu \mathrm{R}$ value shown is for a shear rate of $20 \mathrm{~s}^{-1}$. The data corresponds to the boxed area in Figure 7. The uncertainty was found to be approximately $5 \%$ for the experimental data [4].

Figure 9: Comparison of modeled vs experimental viscosity curves for $20 \%$ composition mortar mixture. Relative viscosity, $\mu \mathrm{R}$, was modeled with respect to the baseline curve. The standard uncertainty for the plastic viscosities of SRM paste is $0.74 \mathrm{~Pa} \cdot \mathrm{s}$ per the SRM 2492 certificate of analysis. 18

Figure 10: Comparison of modeled vs experimental result flow curves for a $40 \%$ composition mortar mixture. Relative viscosity, $\mu \mathrm{R}$, was modeled with respect to the baseline curve. The 
standard uncertainty for the plastic viscosities of SRM paste is $0.74 \mathrm{~Pa} \cdot \mathrm{s}$ per the SRM 2492 certificate of analysis. 18

Figure 11: Diagram of confined coaxial rheometer system shown shearing a general suspension. The purple bar represents the lid created to encapsulate the system and eliminate any free surfaces.

Figure 12: Influence of capping the rheometer increased the relative viscosity for the geometry used but not enough to match the $40 \%$ model data. The standard uncertainty for the plastic viscosities of SRM paste is $0.74 \mathrm{~Pa} \cdot \mathrm{s}$ per the SRM 2492 certificate of analysis. The uncertainty was found to be approximately $5 \%$ for the experimental data [4].

Figure 13: Influence of capping the rheometer increases relative viscosity and exceeds the $20 \%$ model. The standard uncertainty for the plastic viscosities of SRM paste is $0.74 \mathrm{~Pa} \cdot \mathrm{s}$ per the SRM 2492 certificate of analysis. Uncertainty was found to be approximately $5 \%$ for the experimental data from [4]

Figure 14: Various particle sizes reported in the literature yielded various relative viscosities for suspensions similar to this study. 25

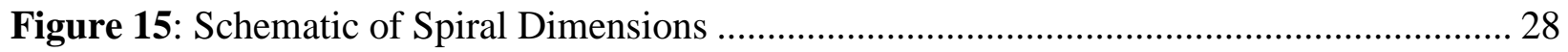

Figure 16: Double Spiral Tools Utilized. See Table 5 for details on the spirals

Figure 17: Paste tests - average results. Uncertainty represents one standard deviation of all measurements with 5 different spirals.

Figure 18: Mortar results averages. Uncertainty represents one standard deviation of all measurements with 5 different spirals 


\section{List of Tables}

Table 1: Simulation data portrayed in Figure 2 as x's and o's. The uncertainty in values of viscosity, based on the standard deviation, is $10 \%$ or lower.

Table 2: Scaling parameters to collapse the mortar curves to the SRM 2492 curve with known concentration. The uncertainty in scaling parameters, based on a least square fit of simulation

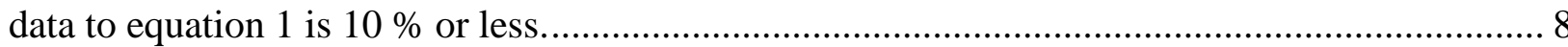

Table 3: Relative viscosity $(\mu \mathrm{R})$ values based on the calibrated experimental data at concentrations of $20 \%$ and $40 \%$ by volume, labeled $\mu R 20$ and $\mu R 40$, respectively. The uncertainty was approximately $5 \%$ for the experimental data [4]. 15

Table 4: The relative viscosity $(\mu \mathrm{R})$ values based on experimental data are compared to the predicted $\mu \mathrm{R}$ from model. The uncertainty was found to be approximately $5 \%$ for the experimental data [4].

Table 5: Comparison of relative viscosity when using a normal ( $\mu R)$ vs confined system $\left(\mu R^{*}\right)$ on mortar samples of $20 \%$ and $40 \%$ volume compositions. Model values are included for comparison. The uncertainty was found to be approximately $5 \%$ for the experimental data [4].21

Table 6: Relative viscosities from the literature for mixtures similar to our 40 \% SRM 2493 mortar. The various particle sizes analyzed in those studies are also displayed. The $1000 \mu \mathrm{m}$ data point from Study XV was not included in Figure 14 for x-axis clarity.

Table 7: Summary of double spiral tools varying in manufacturing characteristics. The nominal tool diameter was $35.00 \mathrm{~mm}$ and the length was $50.00 \mathrm{~mm}$. The uncertainty of the caliper was $0.05 \mathrm{~mm}$ 


\section{Acknowledgments}

The authors would like to thank some key people without whom this study could not have been completed: Max Peltz (NIST), Michael J. Boisclair (NIST), Didier Lootens (SIKA), and John "Rusty" Hettenhouser (NIST). Thanks to Max Peltz for all of the assistance with properly running the equipment at the laboratory. Also, thanks to John "Rusty" Hettenhouser for aiding in the development of the 3D printed spirals and Didier Lootens (SIKA) for providing the original technical drawing.

We would also like to acknowledge that this research used resources of the Argonne Leadership Computing Facility at Argonne National Laboratory, which is supported by the Office of Science of the U.S. Department of Energy under contract DE-AC02-06CH11357. 


\section{Introduction}

Suspensions are found in a wide range of commercial products such as foods, cosmetics, paints, and concrete. A suspension is a system that contains solid particles dispersed in fluid medium [5]. Suspension flow is an important characteristic of many of these products, and rheology is the science used to measure this property. Many rheological models have been developed to predict how changes in a suspension's constituents affect the flow or rheological behavior of the material. For instance, mortar can be represented as a suspension of sand particles in a cement paste medium. The cement paste can be further categorized as being a suspension of cement particles in an aqueous medium, water. Materials such as cement paste or mortar, with its high solids concentration, poly-sized particulates/particles, and time-dependent behavior, have proven to be complex; thus sophisticated models are needed to characterize their rheological behavior. In order to develop these models, it is important to ensure that the cement-based suspension is tested in the appropriate rheometer and that the rheological data obtained from the rheometer are accurate. Most rheometers are designed using an approximation of a Couette geometry. Couette geometry [6] is defined as shearing between two parallel surfaces, one of which is moving relative to the other such that a linearly varying fluid velocity profile develops between the surfaces. The most common geometries used for concrete rheometers are coaxial cylinders and vanes.

To determine the rheological properties of mortar, the gap between the shearing surfaces of a rheometer should be able to accommodate sand of at least $1 \mathrm{~mm}$ in diameter. In this study, spherical glass beads were used to represent the sand, and the rheometer's gap size was typically of order 10 times the particle size. Additionally, slippage and shear-induced migration of particles during measurements are also of concern in conventional rheometers [7]. Suspended particles, no matter how small, experience radial sedimentation or migration away from the rotating surface of the rheometer, even at low shear rates. This effect causes the material sheared between the surfaces to no longer be uniform, which can eventually result in a diluted, lower viscosity layer occurring near the rotating wall. This phenomenon ultimately causes the measured viscosities of the bulk fluid to be lower than the "real" viscosity of the bulk fluid. Roughening the surfaces of the 
rheometer walls or using a vane geometry are two methods that are commonly used to combat slippage [8]. The vane geometry is assumed to make up a cylindrical shearing surface defined by its height and diameter. As the vane rotates, an artificial wall of material is formed between the blades all around its outer edge, and consequently rotates as a rigid cylinder of material minimizing slippage. This is not always correct, as was demonstrated by computer simulations [9], which showed that the material between the blades is not homogeneous and that aggregates have a tendency to migrate away from the vane introducing a "slip" layer near the outer edge of the vane. Additionally, mixer-type geometries (e.g. helical ribbon [10]) can be used in suspensions with large distribution of particles size and have shown to be a good solution for rheological characterization problems like phase separation of basic constituents [11]. However, a disadvantage of using mixer-type rheometer geometries is that the velocity field (and thus shear rate) are ill-defined due to complex flow patterns that may be present [6]. This renders interpretation of the data difficult and introduces the need for a reference material, ideally with characteristics similar to the suspension of interest, to calibrate the rheometer. The need for calibration of rotational rheometers to measure suspensions with particles up to $1 \mathrm{~mm}$ in diameter or more spurred National Institute of Standards and Technology (NIST) to develop a new series of Standard Reference Materials (SRM) that exhibit a non-Newtonian behavior and are suspensions.

In this report, the rheological behaviors of SRM 2492 and SRM 2493, were measured experimentally and the results were compared to predictions provided by computer model simulations of ideal Couette suspension flows. SRM 2492 is a paste-type SRM and it consists of a limestone suspension in an aqueous solution of corn syrup. SRM 2493 is a mortar-type SRM and is made by adding $1 \mathrm{~mm}$ mono-sized spherical glass beads to SRM 2492. The experimental measurements were conducted using a coaxial rotational rheometer and three different spindle geometries were evaluated, and the optimal rheometer design to avoid slippage is discussed. 


\section{Materials \& Procedures}

\subsection{Materials}

The materials used in this study were SRM $2492^{1}$ and SRM $2493^{1}$. SRM 2492 is a standard reference material for paste for rheological studies. It is composed of distilled water, limestone, and corn syrup. The addition of $1 \mathrm{~mm}$ glass spherical beads transforms the paste SRM 2492 into the mortar SRM 2493. The components of SRM 2492 were mixed in a high shear blender following the procedure in ASTM C1738 [12] and as described in the re-certification report [13]. Once the paste was blended, the $1 \mathrm{~mm}$ beads were introduced using a high-speed plunger mixer at 300 RPM (31.4 rad/s). Two concentrations were used for the addition of beads: $20 \%$ and $40 \%$ beads by volume. The time required to make the mixtures homogeneous ranged from 3 min to 5 min. While 3 min was more than sufficient to homogenize the $20 \%$ by (volume mixture), two additional minutes were required for the $40 \%$ mixture. References [13] and [4] give more details about the SRM characteristics.

\subsection{Rheological Measurements}

Measurements for the rheological properties of the materials were conducted using a coaxial rotational rheometer, composed of an outer container (i.e., a cup) and an inner spindle. The outer container was cylindrical, with a diameter of $43 \mathrm{~mm}$ and a height of $80 \mathrm{~mm}$ with serration ribs on its inner wall about $1 \mathrm{~mm}$ in depth and thickness. The inner spindle geometry was varied in design. In this set-up, the outer container was fixed and the inner spindle rotated.

Three different spindle geometries were used in this work, as shown in Figure 1: a solid cylinder with serration (SS18) ${ }^{2}$; a vane-type spindle with 6 blades (RHN-83C); and a doublehelical spiral (RHN-83A). SS18 has a diameter of $18 \mathrm{~mm}$ and length of $55 \mathrm{~mm}$. The serrations on the cylindrical spindle were the same as on the outer cup, i.e., about $1 \mathrm{~mm}$ thick and deep. RHN-

${ }^{1}$ Certificate and instructions can be found at www.nist.gov

${ }^{2}$ Each geometry is complemented with a NIST code and this code will be used periodically throughout this report. 
83C has the same dimensions as the SS18, and it is designed to have six blades. RHN-83A is a mixer-type spindle that has the shape of an uninterrupted double-helical spiral. The diameter of RHN-83A is $35 \mathrm{~mm}$ and its vertical length is $50 \mathrm{~mm}$. The coaxial cylinder and the vane were fabricated from stainless steel. The double spiral was obtained by 3D printing using various plastic as described in section 4.2, where the influence of 3D printing of the spiral on the measurements was compared. [9]

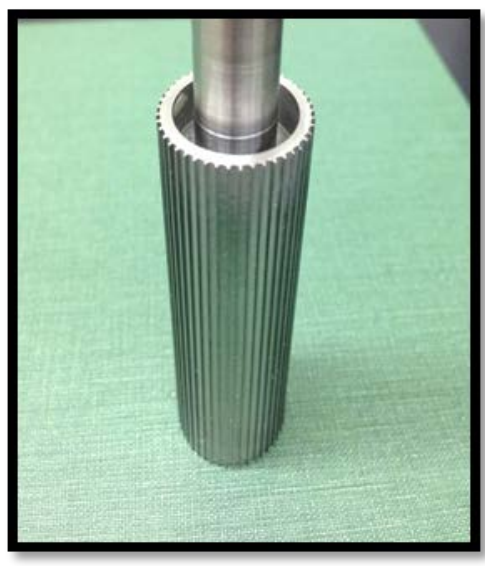

Serrated coaxial cylinder. SS18

$\mathrm{D}=18 \mathrm{~mm}$

$\mathrm{H}=55 \mathrm{~mm}$

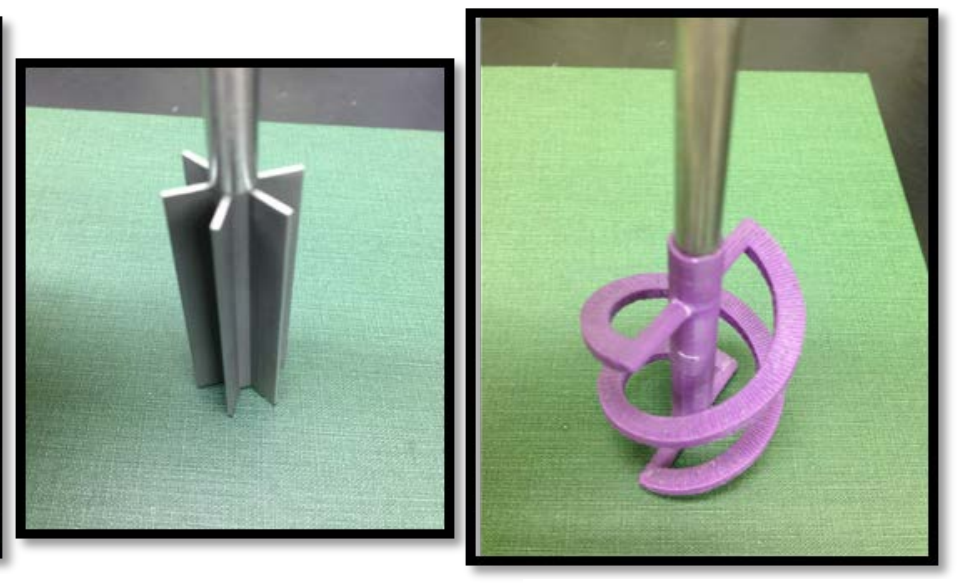

Six-blade vane.

RHN-83C

$\mathrm{D}=18 \mathrm{~mm}$

$\mathrm{H}=55 \mathrm{~mm}$
Double helical spiral.

RHN-83A

$\mathrm{D}=35 \mathrm{~mm}$

$\mathrm{H}=50 \mathrm{~mm}$

Figure 1: Three geometry types used for testing, each representing a different spindle family.

The rheometer used dedicated software to record the imposed rotational speed and respective torque generated by shearing the material of interest. However, to create a flow or viscosity curve for analysis, the raw data required conversion into fundamental units of viscosity [Pa·s] and shear rate $\left[\mathrm{s}^{-1}\right]$. Thus, a method was developed at NIST to convert the torque $[\mathrm{Nm}]$ and rotational speed [rpm] into shear stress $[\mathrm{Pa}]$ and shear rate $\left[\mathrm{s}^{-1}\right]$ by using the SRM 2492 paste as a calibration material, as explained in section 4.3 of the certification report [4]. A spreadsheet [SRM 2493 Data Calibration] was also developed for this procedure, and is posted on the SRM 2493 website ${ }^{3}$.

\footnotetext{
${ }^{3}$ www.nist.gov under Services \& Resources and then select Standard Reference Materials (SRMs). Search for 2493
} 
The measurement protocol consisted of 15 steps of increasing speed ranging from $0.1 \mathrm{rpm}$ $(0.01 \mathrm{rad} / \mathrm{s})$ to $100 \mathrm{rpm}(10.47 \mathrm{rad} / \mathrm{s})$ to generate the "up-curve”, followed by 20 steps of decreasing speed to generate the "down-curve". Each step was held for $30 \mathrm{~s}$. This protocol was selected in order to give the measurements enough time to stabilize and record an accurate torque value. More steps were recorded for the down-curve then for the up-curve in order to gather more data and create a more accurate account of thixotropic behavior, if present. Once the raw data were calibrated the resulting shear rate ranged from $0.03 \mathrm{~s}^{-1}$ to $30.3 \mathrm{~s}^{-1}$ when using the double spiral. The calibrated shear rates differed slightly depending on the rheometer spindle used due to the different inertias imposed while shearing. The calibrated shear rates for the six blade vane and serrated coaxial cylinder ranged from $0.02 \mathrm{~s}^{-1}$ to $20.7 \mathrm{~s}^{-1}$ and $0.03 \mathrm{~s}^{-1}$ to $25.6 \mathrm{~s}^{-1}$, respectively. 


\section{Modeling Approach}

A two-step approach was used to predict the viscosity of the mortar SRM. First, a direct numerical simulation was used that incorporated the paste SRM rheological properties in a computational model of a hard sphere suspension. Once the viscosity of the mortar was calculated for a finite set of shear rates (four in this case), two scaling parameters were determined that mapped the viscosity versus shear rate data of the suspension to the viscosity versus shear rate data of the matrix fluid (SRM paste). To obtain the full mortar viscosity curve, the inverse of this transformation was applied to the matrix viscosity versus shear rate curve. The full description and validation of this scaling procedure is given in Ref. [14].

The computational approach used in this work for modeling suspensions is based on Smooth Particle Hydrodynamics (SPH) [9]. SPH is a Lagrangian formulation of the Navier Stokes equations that has been adapted to model non-Newtonian fluids containing solid inclusions. A full description of this approach is beyond the scope of this work, but it is worth mentioning a few features of this simulation. A Lees-Edwards boundary condition is used to model Couette flow in the simulation cell [15]. This approach allows for the establishment of a Couette-like velocity profile in an infinite periodic system. As a result, wall effects, which could produce an inhomogeneous density variation or an effective slip phenomenon, are avoided. For an applied rate of strain, the volume averaged stress is calculated. The viscosity is then determined by dividing the volume averaged stress by the shear rate. An additional feature in this simulation is that lubrication forces are included to properly model the interactions between solid inclusions when they are in close proximity as the numerical resolution needed to model such effects is too demanding to accomplish using SPH alone. The approach utilized for this work has been validated for a variety of flow scenarios where excellent agreement occurs between analytic solutions of flow fields for non-Newtonian continuum fluids in channel, tube geometries and in experimental measurements of suspensions composed of micrometer sized spheres with different power law matrix fluids in a Couette geometry $[14,16]$. 
Measurements in this work utilized the SRM 2492 paste to serve as the matrix fluid of a suspension composed of mono-sized glass bead inclusions. The matrix fluid is described by the following empirical equation (see Equation 1 ) for viscosity versus shear rate curve:

$$
\mu=\frac{A}{\gamma^{B}}+C
$$

where

$\dot{\gamma} \leq 1 \quad A=16.411 ; \mathrm{B}=0.988 ; \mathrm{C}=9.883$

$\dot{\gamma}>1 \quad A=19.178 ; \mathrm{B}=0.727 ; \mathrm{C}=7.116$

The coefficients A, B, and C were determined by a least square fit of eq. (1) to the paste SRM 2492 data obtained with the parallel plate geometry, with an additional constraint that the viscosity is the same at $\dot{\gamma}=1$. The viscosity $(\mu)$ is then calculated at any given shear rate with equation (1).

Equation 1 was input into the simulation code to serve as the matrix fluid. The glass beads were modeled as spherical inclusions with 472 and 944 spheres used to model the $20 \%$ and $40 \%$ suspensions, respectively. The uncertainty of the simulated suspension viscosity was derived from calculating the standard deviation of stress values, which is proportional to viscosity, over five to ten values of strain. Four different shear rates were used in the simulation and the viscosity at each shear rate was determined (see Table 1). When the simulation data were rescaled, they fell on top of the viscosity versus shear rate curve of the matrix fluid. The scaling parameters, shown in Table 2 , are then used to generate predictive curves of the suspension's viscosity vs shear rate, as shown in Figure 2, for the $20 \%$ and $40 \%$ volume fraction suspensions. In other words, to produce the scaled predictive curves, the scaling parameter, $\mu_{\mathrm{sc}}$, is factored into the viscosity variable, $\mu$, in equation (1). Similarly, the scaling parameter, $\gamma_{\mathrm{sc}}$, is factored into the shear rate variable, $\dot{\gamma}$, in equation (1). 
Table 1: Simulation data portrayed in Figure 2 as x's and o's. The uncertainty in values of viscosity, based on the standard deviation, is $10 \%$ or lower.

\begin{tabular}{|c|c|c|c|}
\hline $\begin{array}{l}\text { Shear Rate } \\
{[1 / \mathrm{s}]}\end{array}$ & $\begin{array}{c}\text { 0\% Simulated Viscosity } \\
{[\mathrm{Pa}-\mathrm{s}]}\end{array}$ & $\begin{array}{c}\text { 20\% Simulated Viscosity } \\
{[\mathrm{Pa}-\mathrm{s}]}\end{array}$ & $\begin{array}{c}\mathbf{4 0 \%} \text { Simulated Viscosity } \\
{[\mathrm{Pa}-\mathrm{s}]}\end{array}$ \\
\hline 0.1 & 175 & 214 & 297 \\
\hline 1 & 27.4 & 39.4 & 65 \\
\hline 10 & 10.6 & 18.1 & 59 \\
\hline 100 & 7.76 & 14.1 & 56 \\
\hline
\end{tabular}

Table 2: Scaling parameters to collapse the mortar curves to the SRM 2492 curve with known concentration. The uncertainty in scaling parameters, based on a least square fit of simulation data to equation 1 is $10 \%$ or less.

\begin{tabular}{|c|c|c|}
\hline Concentration [\%] & $\mu_{\text {sc }}$ factor & $\dot{\gamma}_{\text {sc }}$ factor \\
\hline 20 & 1.85 & 0.66 \\
\hline 40 & 7.2 & 0.23 \\
\hline
\end{tabular}




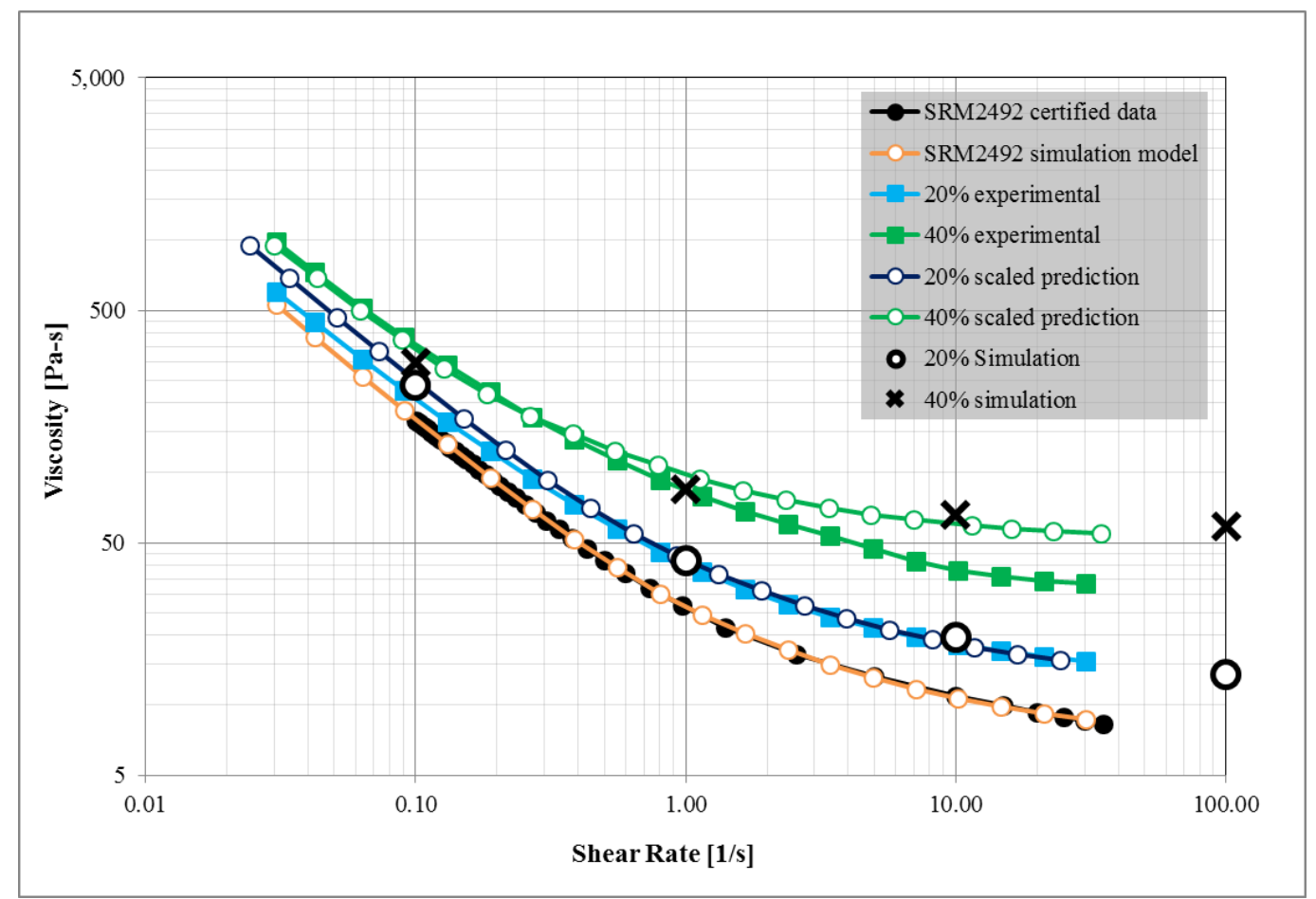

Figure 2: Simulation model data and predicted data from scaling parameters are compared to experimental data results. Uncertainty was found to be approximately $5 \%$ for the experimental data [4].

In Figure 2, the SRM 2492 paste's certified data baseline is shown as a solid black line. Also displayed are the simulation data for $20 \%$ volume fraction and $40 \%$ volume fraction. The simulation data for the $20 \%$ volume fraction are represented with open circles in Figure 2, whereas data for the $40 \%$ volume fraction are represented using an $\mathrm{X}$. The scaled prediction lines are the curves produced by rescaling the certified data with the scaling parameters. The scaling parameters that produced those curves are given in Table 2, which are based on numerical simulations of a suspension composed of mono-size spheres in a matrix fluid that has a viscosity versus shear rate dependence given in equation (1) and closely matches that of the SRM 2492 paste. As can be seen in Figure 2, there is excellent agreement between the $20 \%$ experimental data and predictions based on the $20 \%$ simulations. However, the $40 \%$ data only agree well with the scaled prediction at the low shear rates, having a noticeably lower value of viscosity at the high shear rates. This lower viscosity, found in the experimental data, is believed to be due to enhanced slip near the 
vane blades and a possible migration of the glass beads outwards from the vane blades as the solid concentration increases.

\subsection{Experimental results}

The rotational speed and torque generated were recorded for the three rheometer geometries selected using both the paste SRM 2492 and the mortar SRM 2493. The first step was to measure SRM 2492 with the various rheometer spindles selected in this study and to compare the results with the certified data, as shown by the viscosity curve comparison in Figure 3. To calibrate the rheometer with respect to each spindle used, the raw viscosity vs. shear rate curves for each of the three spindle were scaled to match the SRM 2492 certified data obtained using a parallel plate rheometer geometry [13]. Figure 3 shows that the calibrated curves closely trace the reference curve. The reference curve is the certified SRM 2492 data (shown with a black curve). The standard uncertainty for the plastic viscosities of SRM paste is 0.74 Pa.s per the SRM 2492 certificate of analysis. The orange shaded box shown in Figure 3 highlights the shear rates that were not used during SRM 2492 paste certification protocol, but were included for the SRM 2493 mortar certification to observe the behavior of the paste at lower shear rates; thus, the SRM 2492 curve does not extend below $0.1 \mathrm{~s}^{-1}$. The behavior of the SRM 2492 paste when subjected to low shear rates is portrayed more clearly in Figure 4. Similarly, the blue and red shaded areas portraying the mid-range and high-range behavior are shown more clearly in Figure 5 and Figure 6, respectively. 


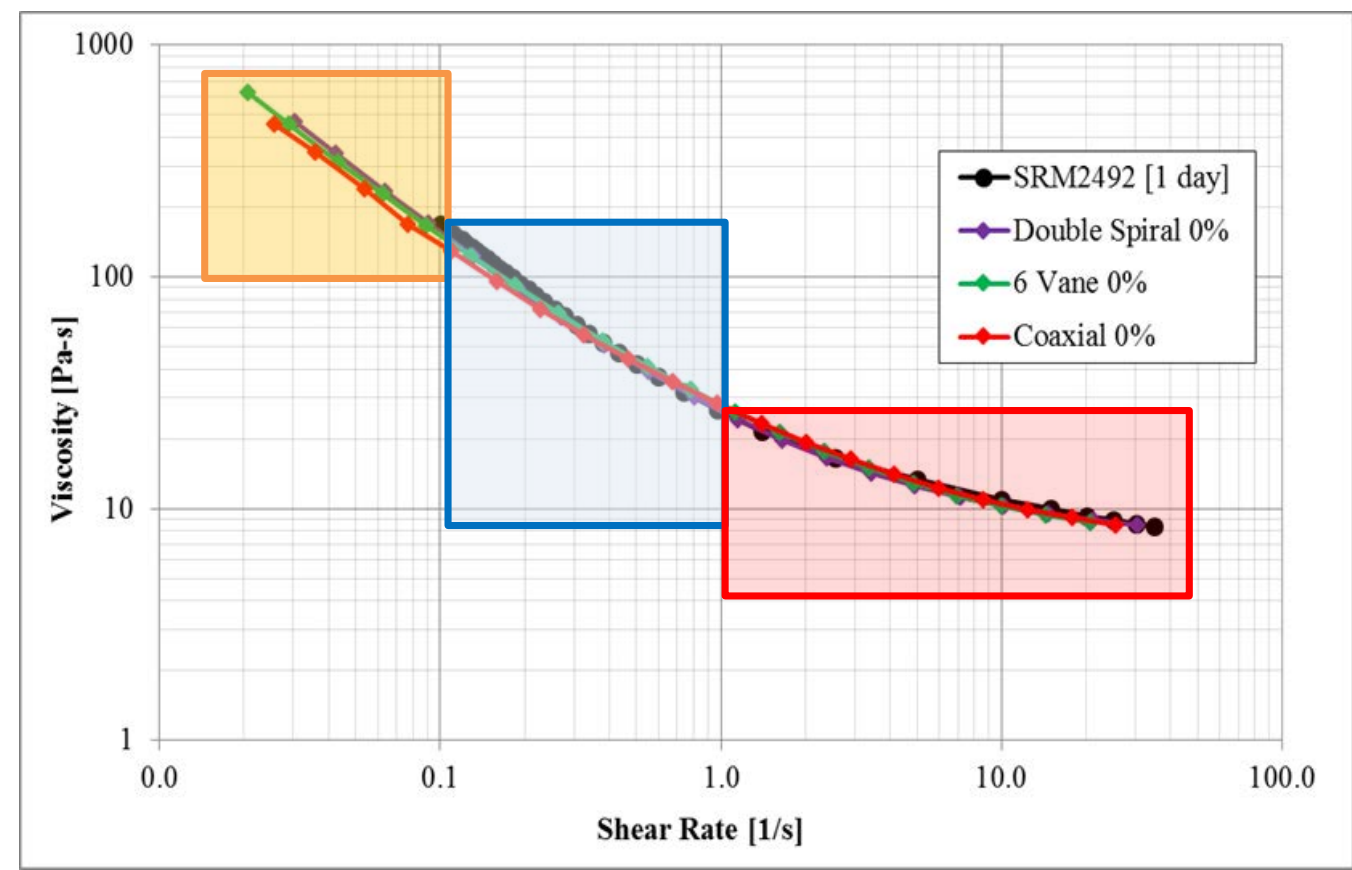

Figure 3: Viscosity curve comparison of the calibrated experimental data when SRM paste ( $0 \%$ beads) was sheared using all three spindles. The flow curves are compared to their reference curve shown in black, which represents the SRM2492 certified values. Uncertainty was found to be approximately $5 \%$ for the experimental data from [4].

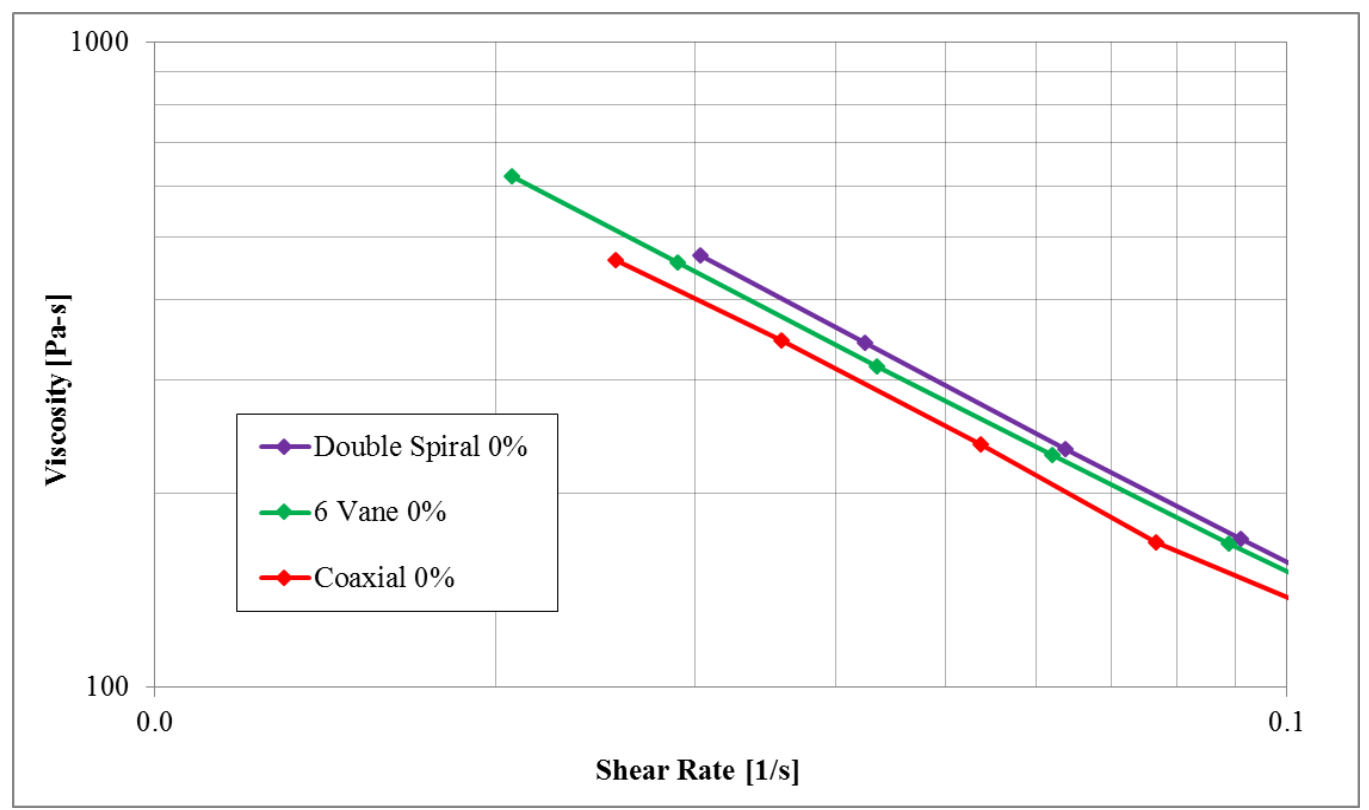

Figure 4: Behavior of SRM paste at low-range shear rates $\left(<0.1 \mathrm{~s}^{-1}\right)$, corresponding to the orange-shaded region in Figure 3. Uncertainty was found to be approximately $5 \%$ for the experimental data from [4]. 


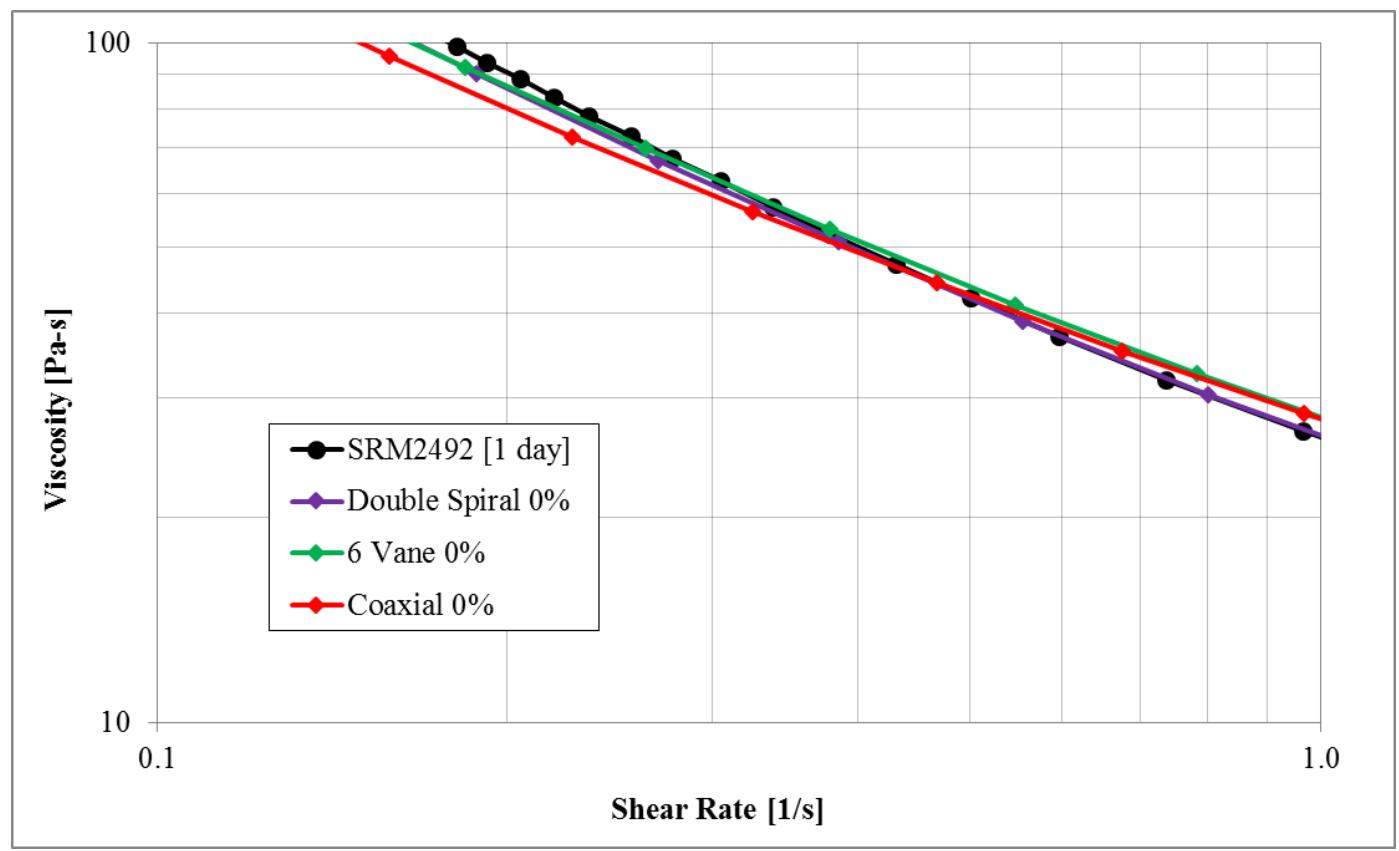

Figure 5: Behavior of SRM paste at mid-range shear rates $\left(0.1 \mathrm{~s}^{-1}<1.0 \mathrm{~s}^{-1}\right)$, corresponding to the blue-shaded area from Figure 3 . Uncertainty was found to be approximately $5 \%$ for the experimental data from [4]. 


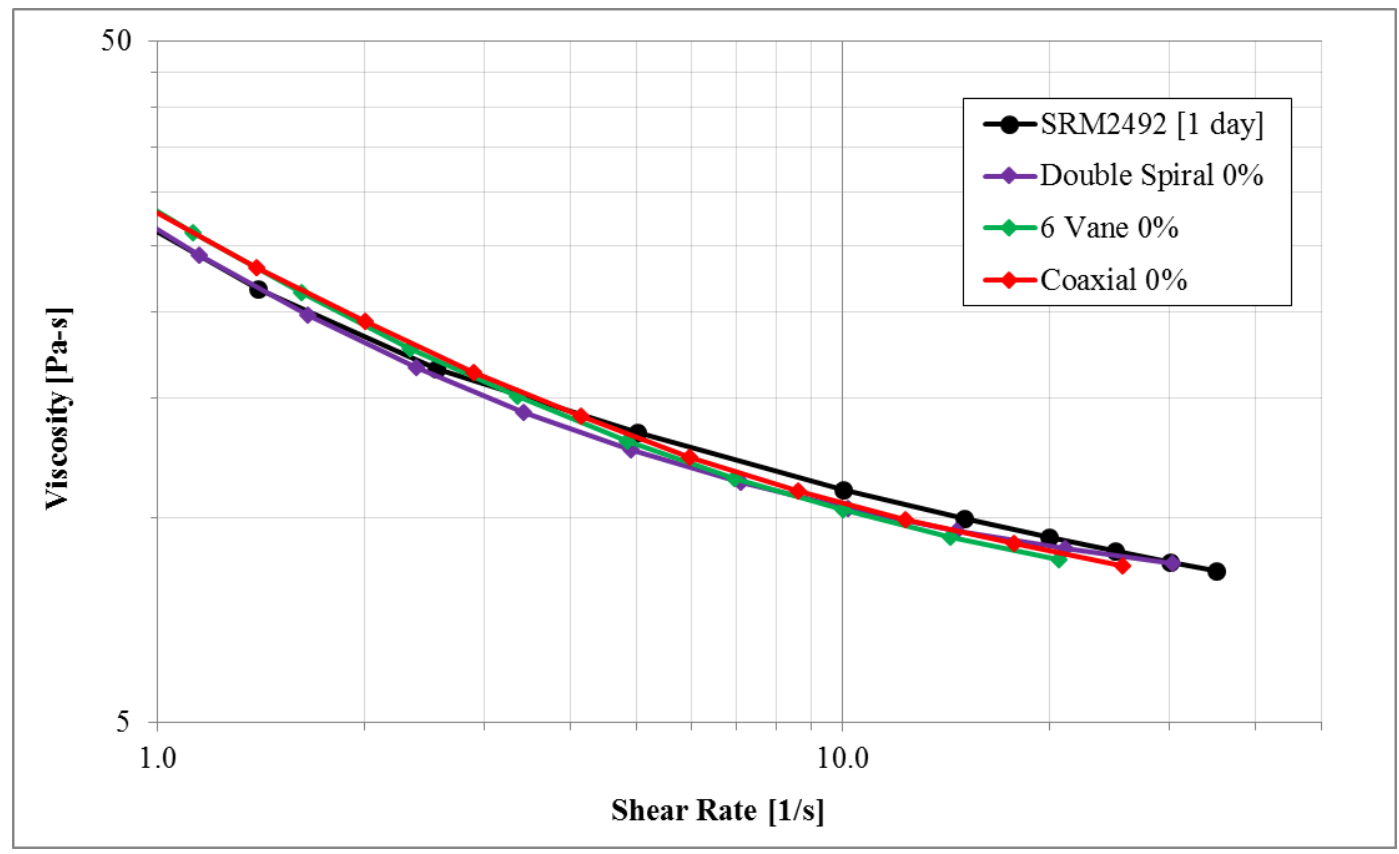

Figure 6: Behavior of SRM paste at high-range shear rates $\left(1.0 \mathrm{~s}^{-1}<50 \mathrm{~s}^{-1}\right)$, corresponding to the red shaded area from Figure 3 . The uncertainty was found to be approximately $5 \%$ for the experimental data from [4].

All the experimental data obtained in this study are summarized in Figure 7, where the three rheometer spindles were all tested on a mortar reference material (SRM 2493) of $20 \%$ and $40 \%$ by volume bead concentrations. The graph compares all experimental viscosity curves to the certified SRM 2492 curve, which was the reference baseline for this study. The difference in viscosities between the baseline and any other viscosity curve of interest is considered the relative viscosity $(\mu \mathrm{R})$. The relative viscosity is a factor that represents the effects on viscous behavior caused by using different spindles and material sets (i.e. bead concentration). Figure 8 shows an example of $\mu \mathrm{R}$, based on the double spiral spindle and $40 \%$ mortar concentration.

It is evident from the data shown in Figures $\mathbf{7}$ and $\mathbf{8}$ that relative viscosity depends on the spindle used for performing the rheological measurement. The relative viscosity values show a decreasing trend from double spiral to six blade vane and serrated coaxial cylinder, respectively. The trend signifies that the different spindles vary in effectiveness of shearing. This effectiveness can be correlated to how well the rotating surface of the measurement system is actually able to 
grasp the material and shear it without slippage. Any slippage (non-effective shearing) results in reducing the relative viscosity. This effect is more evident at higher bead concentrations, which is shown when comparing the $40 \%$ curves in Figure 7 to the $20 \%$ curves. In the next section, the experimental viscosity curves are compared to the modeled (predicted) curves.

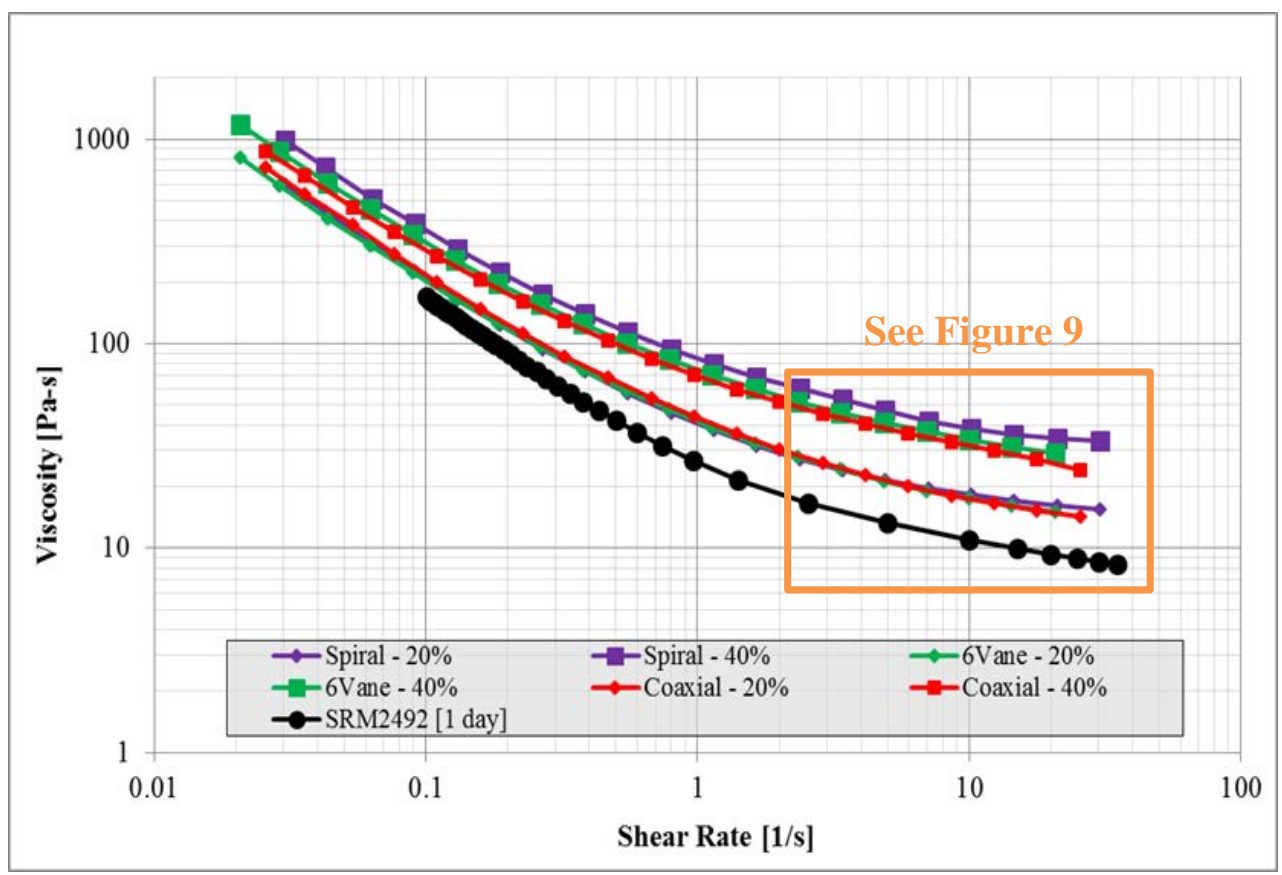

Figure 7: Viscosity curve at concentrations of $20 \%$ and $40 \%$, by volume, of glass beads. The uncertainty was estimated to be $10 \%$ of the viscosity. Full discussion on the uncertainty can be found in the full report $[13,4]$. 


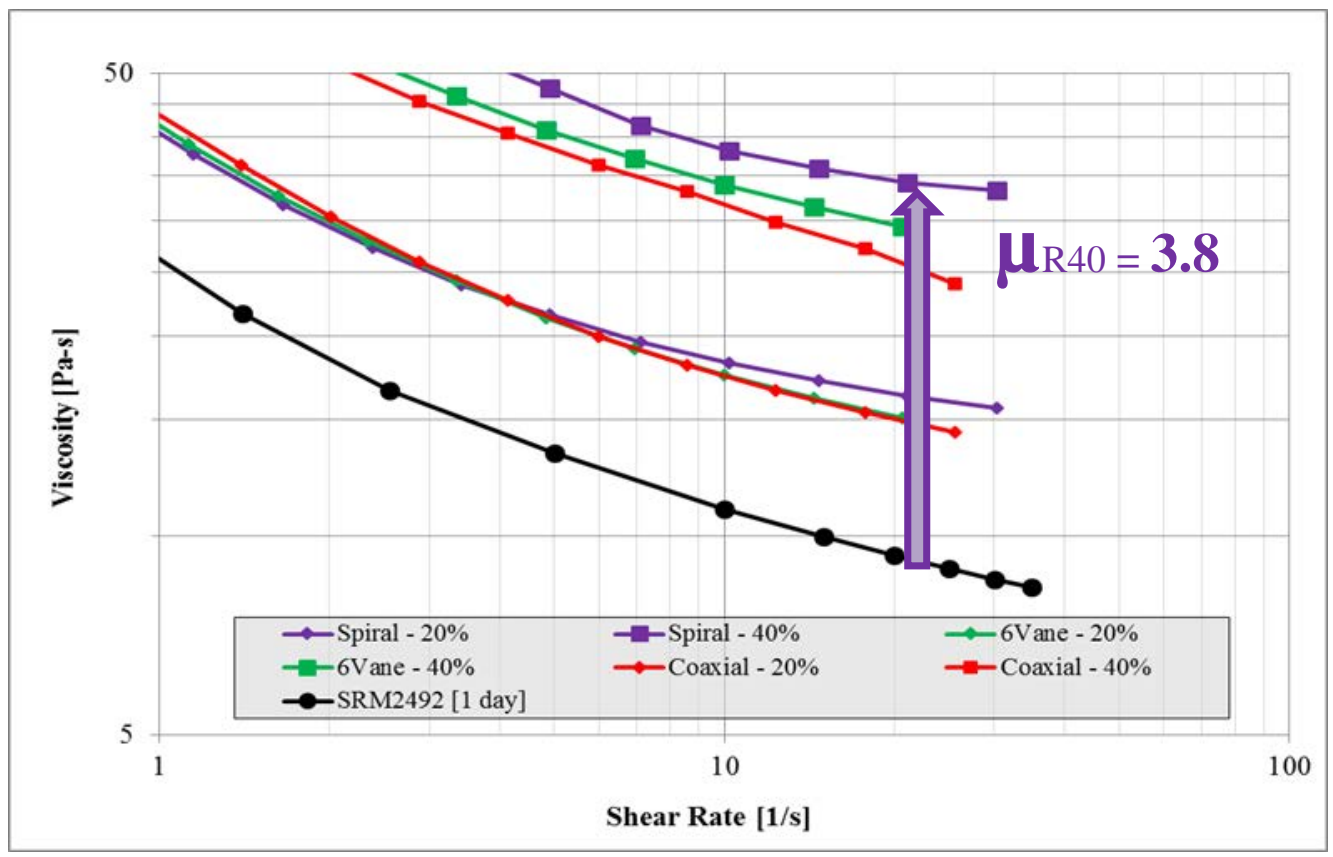

Figure 8: One example of relative viscosity $(\mu \mathrm{R})$, for double spiral and a material set of $40 \%$ beads by volume. The $\mu \mathrm{R}$ value shown is for a shear rate of $20 \mathrm{~s}^{-1}$. The data corresponds to the boxed area in Figure 7. The uncertainty was found to be approximately $5 \%$ for the experimental data [4].

Figure 8 provides a closer look at the differences in viscosity by displaying the $\mu \mathrm{R}$ value for the $40 \%$ bead volume sample prepared using the spiral. To avoid congesting the graph, Figure 8 does not portray all the $\mu \mathrm{R}$ values calculated in this study. Rather, the relative viscosity for all spindle/material combinations are displayed in Table 3 at three shear rates. The three shear rates were selected to portray the relative viscosity at low, medium, and high shear rates. It is evident

Table 3: Relative viscosity $(\mu \mathrm{R})$ values based on the calibrated experimental data at concentrations of $20 \%$ and $40 \%$ by volume, labeled $\mu R 20$ and $\mu R 40$, respectively.

The uncertainty was approximately $5 \%$ for the experimental data [4].

\begin{tabular}{|c|c|c|c|c|c|c|}
\hline \multirow{2}{*}{$\begin{array}{c}\text { Shear Rate } \\
{[1 / \mathrm{s}]}\end{array}$} & \multicolumn{2}{|c|}{ Double Spiral } & \multicolumn{2}{c|}{ 6 Blade Vane } & \multicolumn{2}{c|}{ Coaxial Cylinder } \\
\cline { 2 - 7 } & $\mu \mathrm{R} 20$ & $\mu \mathrm{R} 40$ & $\mu \mathrm{R} 20$ & \multicolumn{1}{c|}{$\mu \mathrm{R} 40$} & $\mu \mathrm{R} 20$ & $\mu \mathrm{R} 40$ \\
\hline $\mathbf{0 . 1}$ & 1.3 & 2.3 & 1.3 & 2.0 & 1.6 & 2.1 \\
$\mathbf{1}$ & 1.6 & 3.3 & 1.5 & 2.7 & 1.5 & 2.5 \\
$\mathbf{2 0}$ & 1.8 & 3.8 & 1.7 & 3.4 & 1.7 & 2.8 \\
\hline
\end{tabular}


from the table that $\mu \mathrm{R}$ values portray a major difference caused by two factors: shear rate and bead concentration. First, when the mortar tested had a high concentration of glass beads ( $40 \%$ by volume), the relative viscosity ( $\mu \mathrm{R} 40$ ) differs greatly depending on the spindle used. When testing the lower glass bead concentration material ( $20 \%$ by volume), the relative viscosities ( $\mu$ R20) do not differ significantly. The second noticeable trend is caused by the shear rate. At high shear rates, major differences also exist, based on the spindle used, yet at low shear rates the relative viscosities are nearly identical.

\subsection{Model results}

The viscosity curve model was created with the intention of predicting the viscous behavior dependence on the material's composition. The baseline for the model was created using the NIST certified data for SRM 2492, and algorithms [14] developed to predict the change in viscous behavior with respect to the baseline curve due to the addition of beads in different concentration.

Figure 9 portrays the $20 \%$ predicted (computer modeled) viscosity curve and its relative viscosity $(\mu \mathrm{R})$ in order to compare with the experimental relative viscosities. Similarly, the $40 \%$ curves are shown in Figure 10. These modeled curves are the same "predicted" curves from Figure 2 , which were developed using the scaling factors from Table 2 . The relative viscosities at the high shear rates are good comparison points for a quantitative analysis between experimental and model curves. The $\mu \mathrm{R}$ at low shear rates were omitted in the figures since [in section 3.1] the high shear rates were found to be the cause of any major differences. However, Table 4 displays the measured values of $\mu \mathrm{R}$ at low, medium, and high shear rates and compares them to the predicted $\mu \mathrm{R}$ values from the model. As shown, the model predicted the viscosity to be higher than the resulting experimental data for all three spindles used. This difference is more evident for $\mu \mathrm{R} 40$ than $\mu \mathrm{R} 20$. However, it should be noted that, once again, the same trend exists between the three types of spindles and highlights that the double spiral is the most accurate when compared to the prediction model. The least accurate proved to be the serrated coaxial cylinder. 
Table 4: The relative viscosity $(\mu \mathrm{R})$ values based on experimental data are compared to the predicted $\mu \mathrm{R}$ from model. The uncertainty was found to be approximately $5 \%$ for the experimental data [4].

\begin{tabular}{|c|c|c|c|c|c|c|c|c|}
\hline \multirow{2}{*}{$\begin{array}{c}\text { Shear Rate } \\
{[1 / s]}\end{array}$} & \multicolumn{2}{|c|}{ Double Spiral } & \multicolumn{2}{|c|}{6 Blade Vane } & \multicolumn{2}{|c|}{ Coaxial Cylinder } & \multicolumn{2}{|c|}{ Model } \\
\hline & $\mu_{\mathrm{R} 20}$ & $\mu_{\mathrm{R} 40}$ & $\mu_{\mathrm{R} 20}$ & $\mu_{\mathrm{R} 40}$ & $\mu_{\mathrm{R} 20}$ & $\mu_{\mathrm{R} 40}$ & $\mu_{\mathrm{R} 20}$ & $\mu_{\mathrm{R} 40}$ \\
\hline 0.1 & 1.3 & 2.3 & 1.3 & 2.0 & 1.6 & 2.1 & 1.3 & 2.0 \\
\hline 1 & 1.6 & 3.3 & 1.5 & 2.7 & 1.5 & 2.5 & 1.5 & 3.8 \\
\hline 20 & 1.8 & 3.8 & 1.7 & 3.4 & 1.7 & 2.8 & 1.7 & 6.1 \\
\hline
\end{tabular}




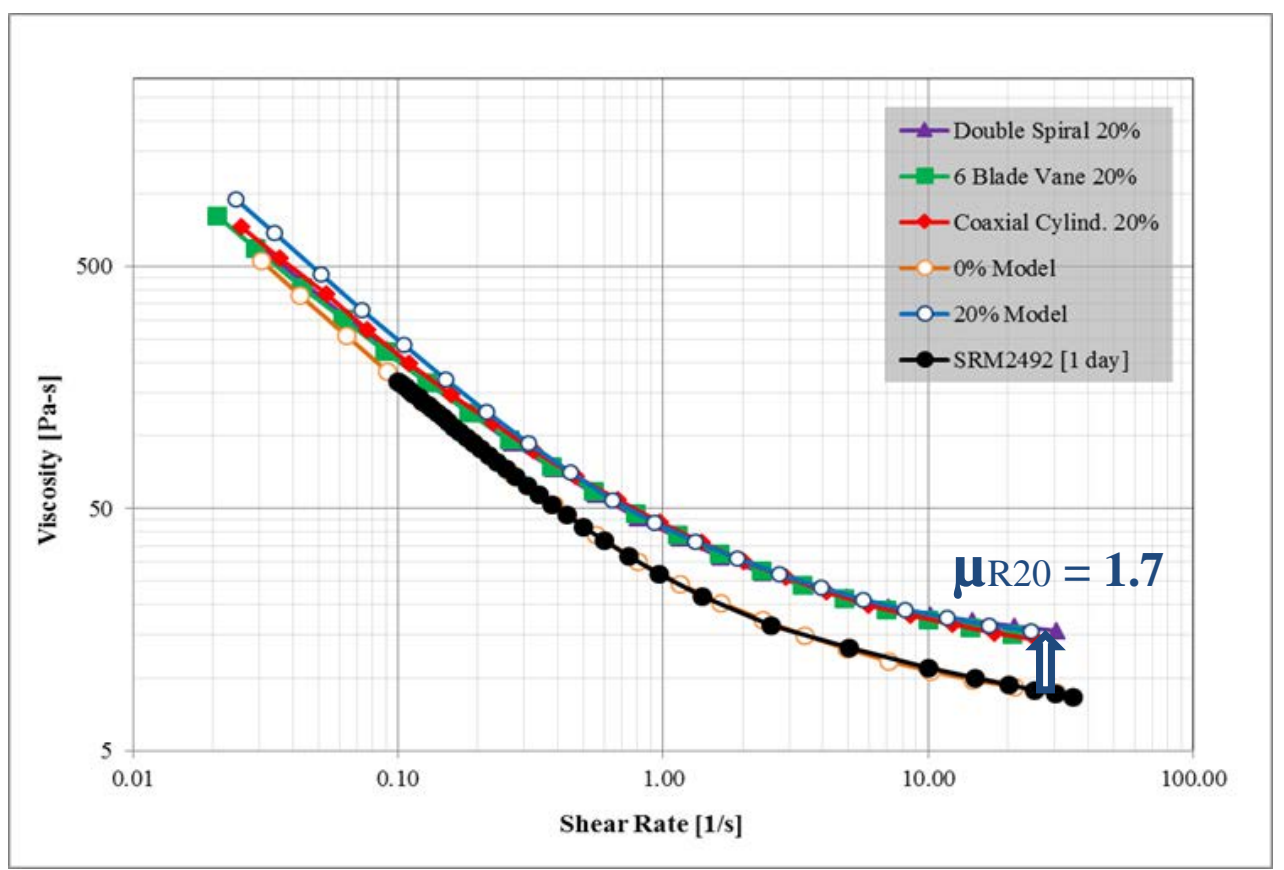

Figure 9: Comparison of modeled vs experimental viscosity curves for $20 \%$ composition mortar mixture. Relative viscosity, $\mu R$, was modeled with respect to the baseline curve. The standard uncertainty for the plastic viscosities of SRM paste is $0.74 \mathrm{~Pa} \cdot \mathrm{s}$ per the SRM 2492 certificate of analysis.

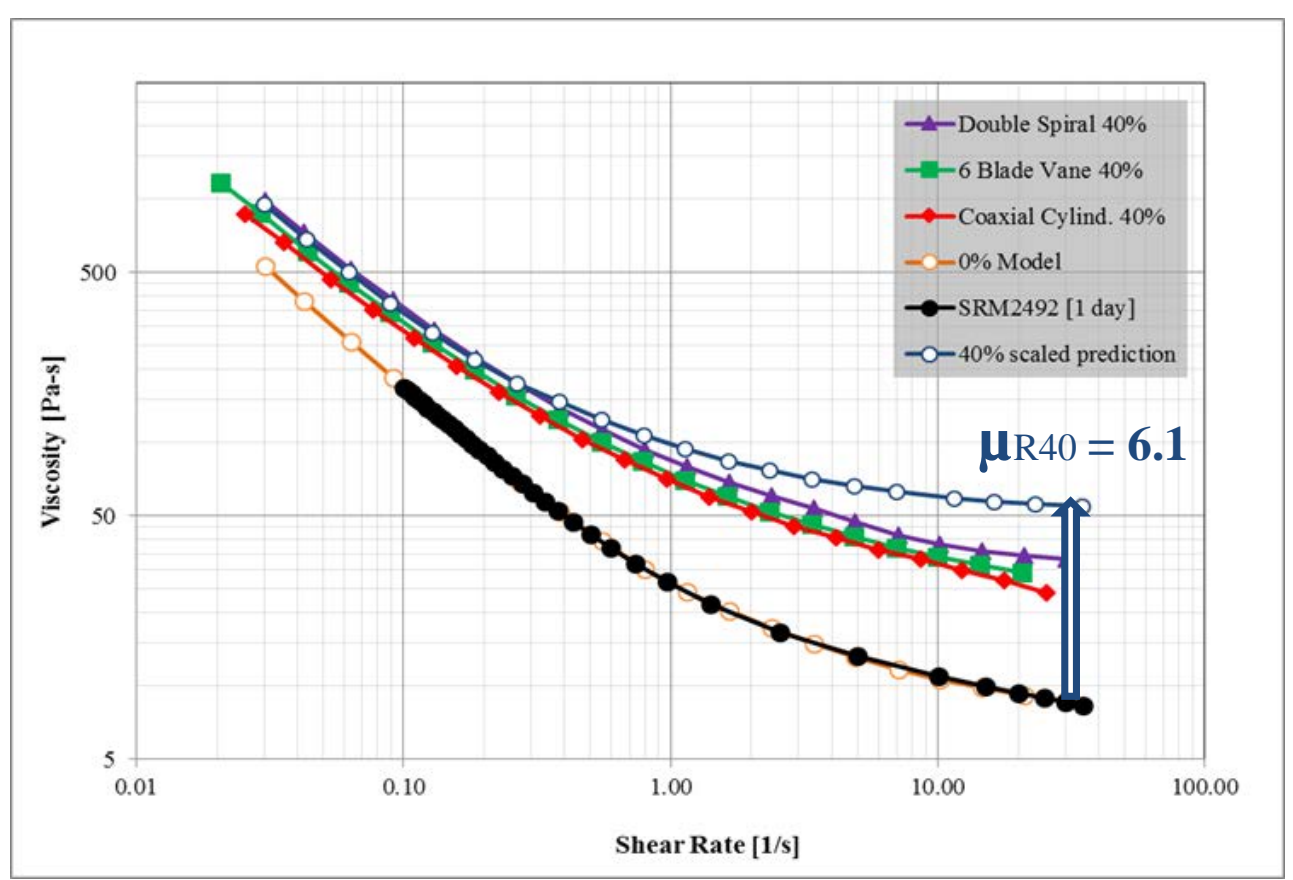

Figure 10: Comparison of modeled vs experimental result flow curves for a $40 \%$ composition mortar mixture. Relative viscosity, $\mu R$, was modeled with respect to the baseline curve. The standard uncertainty for the plastic viscosities of SRM paste is $0.74 \mathrm{~Pa} \cdot \mathrm{s}$ per the SRM 2492 certificate of analysis. 


\subsection{Confined rheological measurements}

The observed reduced viscosity between the experimental data and the simulated data was partially attributed to dilatancy, i.e., the top free surface of the material would rise while the spindle is rotating. Thus, to verify that this phenomenon would have an effect on the measured viscosity by reducing the torque measured, a rheometer system was designed to encapsulate the cup and spindle. As shown in Figure 11, a custom lid was designed and fabricated using a 3D printer at NIST in order to enclose the material and restrict the rheometer system boundaries. This modification eliminated free surfaces, so that the material being sheared would simulate the model's boundary conditions more accurately.

Tests were performed with the enclosed system on samples of $20 \%$ and $40 \%$ mortar compositions by volume fraction, as shown in Figure 12 and Figure 13, respectively. The enclosed lid testing was conducted after the certification testing, but on the same day in order to use the same material. This enclosed testing was only performed on two spindles, double spiral and six blade vane. The confinement modification increased the measured viscosity of the suspensions likely due to the elimination of the free surface at the top. Once the free boundary was no longer present, the fluid did not have the freedom to be displaced upward. Instead, the particles were forced to encounter the lid (restricted top surface), which increases the forces present within the system. This phenomenon was more evident at higher shear rates. Furthermore, the addition of the lid could also be adding a frictional element, which could contribute to the viscosity increase.

The influence of enclosing the rheometer was analyzed by comparing the normal relative viscosity $(\mu R)$ from the previous section (see Table 4 ) to the new relative viscosity (indicated by $\mu R^{*}$ ) found when using the confined system at the three shear rates discussed formerly, as displayed in Table 5.

As mentioned in the previous section, the high-end shear rates $\left(20 \mathrm{~s}^{-1}\right)$ are of interest for discussion in this study since it is the range that is affected the most by changes in mortar composition or rheometer design. Thus, Figures 12 and 13 only portray the results at $20 \mathrm{~s}^{-1}$ (arrow), but the remaining results at lower shear rates are shown in Table 5. The highest impact on relative viscosity due to using a confined system occurred when using the double spiral on a 40 
\% mortar, but even that combination only reached a value 4.5 at $20 \mathrm{~s}^{-1}$, which is less than the simulated value $\mu \mathrm{R}$ of 7.2. However, the confined system did increase the relative viscosity from 3.8 to 4.5 , which shows that using a confined system indeed increases viscosity significantly at 40 $\%$ volume concentration.

On the other hand, the $20 \%$ curve using a confined system resulted in experimental data that was slightly higher than the model $20 \%$ curve when using the double spiral, as shown in Figure 13. Obviously, as the vertical movement of material was prevented by a cap, other artifacts could have been introduced such as friction of the material on the cap. Such effects could be the cause of readings that suddenly spike up, like in the case of the Confined 6 Blade Vane (Conf. 6V - 40 $\%$ on Figure 13) where a few peak points are seen. Thus, although the encapsulation setup at 40 $\%$ concentration showed an increase in the viscosity toward the theoretical estimations, the study concluded that the ideal Couette scenario is not always reproducible in current industry rheometers, especially as volume fraction is increased above $20 \%$. 


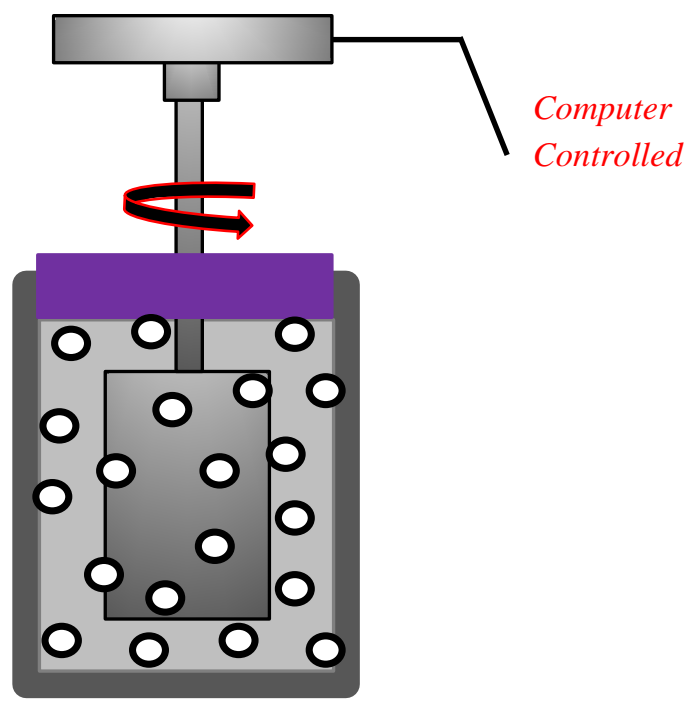

Figure 11: Diagram of confined coaxial rheometer system shown shearing a general suspension. The purple bar represents the lid created to encapsulate the system and eliminate any free surfaces.

Table 5: Comparison of relative viscosity when using a normal $(\mu R)$ vs confined system $\left(\mu R^{*}\right)$ on mortar samples of $20 \%$ and $40 \%$ volume compositions. Model values are included for comparison. The uncertainty was found to be approximately $5 \%$ for the experimental data [4]

\begin{tabular}{|c|c|c|c|c|}
\hline & \multicolumn{3}{|c|}{ Shear Rate $[1 / \mathrm{s}]$} \\
\hline & & 0.1 & 1 & 20 \\
\hline \multirow{4}{*}{$\begin{array}{c}\text { Double } \\
\text { Spiral }\end{array}$} & $\mu_{\mathrm{R} 20}$ & 1.3 & 1.6 & 1.8 \\
\hline & $\mu_{\mathrm{R} * 20}$ & 1.6 & 1.9 & 2.1 \\
\hline & $\mu_{\mathrm{R} 40}$ & 2.3 & 3.3 & 3.8 \\
\hline & $\mathbf{H R}^{*} \mathbf{4 0}$ & 2.5 & 3.5 & 4.5 \\
\hline \multirow{4}{*}{$\begin{array}{c}6 \text { Blade } \\
\text { Vane }\end{array}$} & $\mu_{\mathrm{R} 20}$ & 1.3 & 1.5 & 1.7 \\
\hline & $\mu_{\mathrm{R} * 20}$ & 1.5 & 1.8 & 2.0 \\
\hline & $\mu_{\mathrm{R} 40}$ & 2.0 & 2.7 & 3.4 \\
\hline & $\mu_{\mathrm{R}}^{* 40}$ & 2.2 & 3.1 & 3.6 \\
\hline \multirow{2}{*}{ Model } & $\mu \mathrm{R} 20$ & 1.4 & 1.6 & 1.7 \\
\hline & HR40 & 2.0 & 3.8 & 6.1 \\
\hline
\end{tabular}




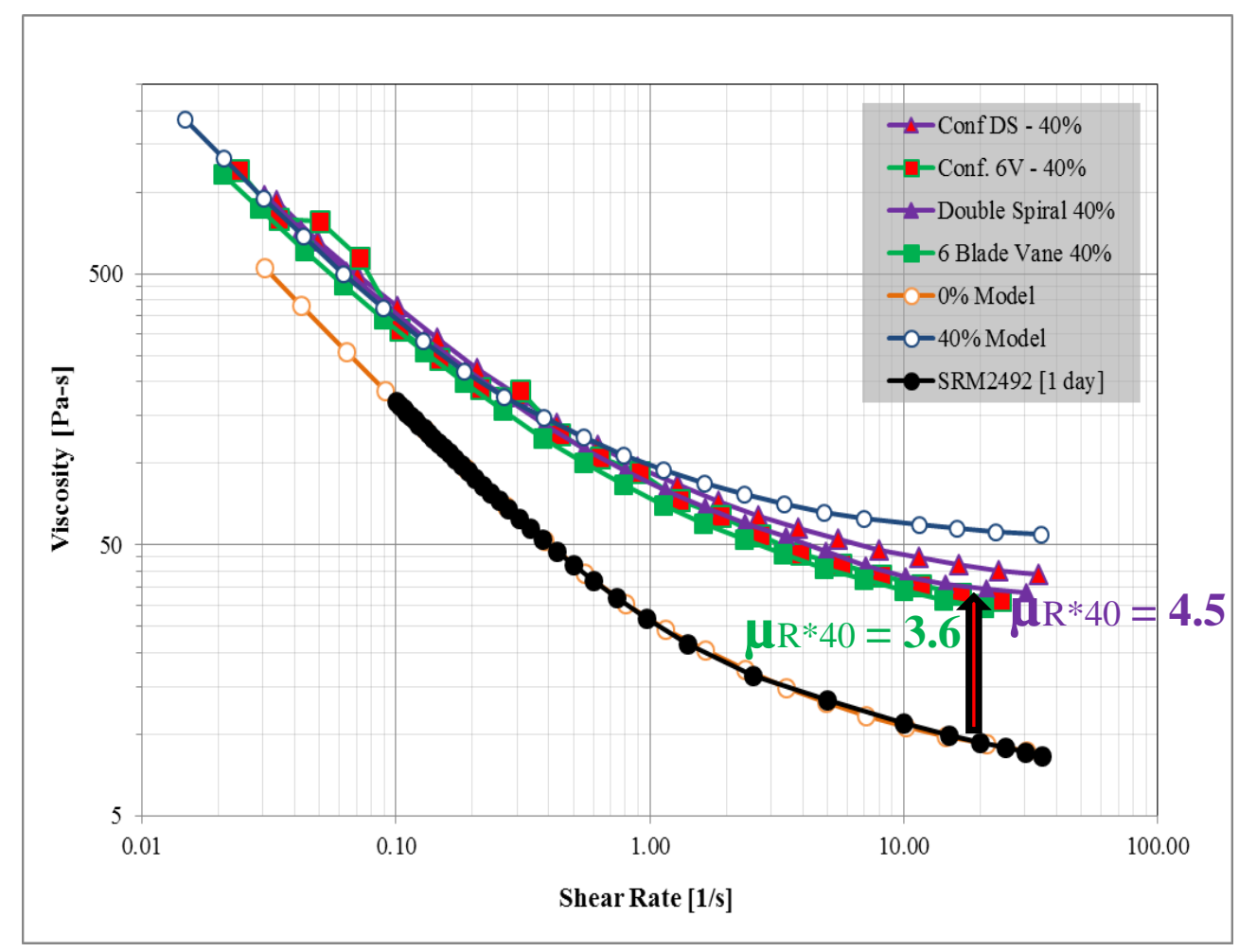

Figure 12: Influence of capping the rheometer increased the relative viscosity for the geometry used but not enough to match the $40 \%$ model data. The standard uncertainty for the plastic viscosities of SRM paste is 0.74 Pa·s per the SRM 2492 certificate of analysis. The uncertainty was found to be approximately $5 \%$ for the experimental data [4]. 


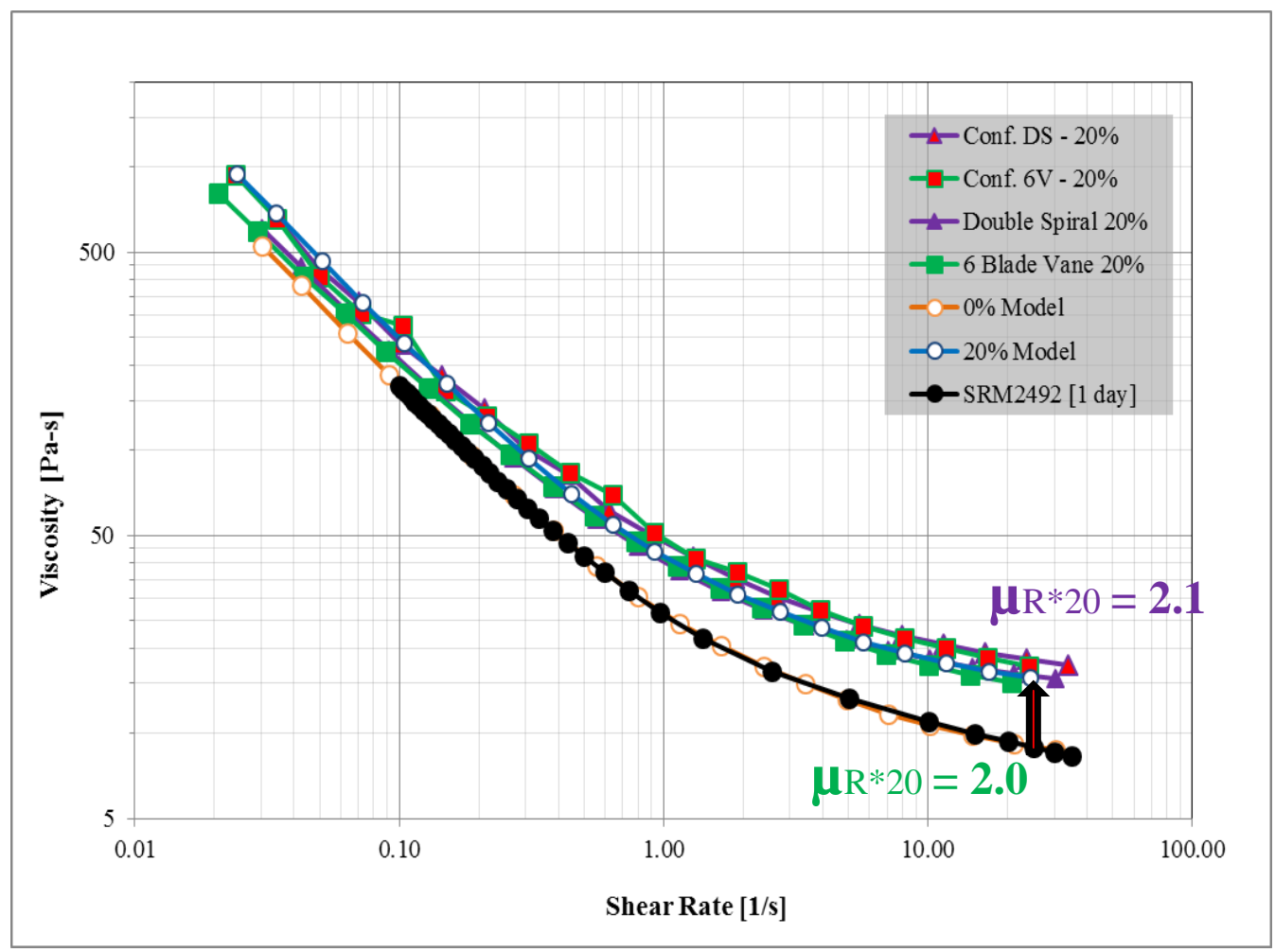

Figure 13: Influence of capping the rheometer increases relative viscosity and exceeds the $20 \%$ model. The standard uncertainty for the plastic viscosities of SRM paste is $0.74 \mathrm{~Pa} \cdot \mathrm{s}$ per the SRM 2492 certificate of analysis. Uncertainty was found to be approximately $5 \%$ for the experimental data from [4].

\subsection{Literature review}

The data gathered from this report were extensively compared to that found in the literature. No significant discrepancy was determined to exist between existing literature and this report when the same type of material was considered, as shown in this section. However, there was a gap in the literature regarding studies dealing with mortar-scale suspensions. Recall the SRM 2492 paste is itself a suspension, composed of a corn syrup solution with limestone powder as the suspended particles. Then, the creation of the mortar SRM 2493 occurs by adding $1 \mathrm{~mm}$ glass beads as the suspended particles in a paste matrix, which increases the suspension complexity. A lack of existing literature for suspensions with similar size inclusions, in cement-type matrices, inspired the creation of this report. 
The limitation of most work in the field of suspension rheology is a tendency to use particles much smaller than those used in concrete systems (sand, $\sim 1 \mathrm{~mm}$ coarse aggregates, $>5 \mathrm{~mm}$ ). One reason for this is that these smaller size are easier to model. For example, more data points can be obtained in a fixed space of modeled simulations by scaling down the size of suspension particles. By reducing the particle size simulated, not only can more particles be modeled, but the data can also be produced in a shorter time. The problem with this approach is that these same models cannot be applied to concrete because the particles are too small to be representative of sand or coarse aggregates used in concrete.

The particle size of the inclusion must be similar in scale $(1 \mathrm{~mm})$ to the inclusions in mortar in order to realistically model mortar. Table 6 compares the maximum particle size of several studies in the literature that related to suspension rheology. The studies presented were those that had a similarity to our study in either relative viscosity values or the volume concentration of suspended particles. Many of these studies analyzed an extended range of volume concentrations. The relative viscosities presented are those which were near $40 \%$. The closest maximum particle size to our study is $1000 \mu \mathrm{m}$ by Reference Study XV in the table. In Study XV, particles ranging from 600 $\mu \mathrm{m}$ to $1000 \mu \mathrm{m}(1 \mathrm{~mm})$ at a $35 \%$ volume concentration were examined, but this study yielded a relative viscosity of only 1.6, as shown in Table 6. The authors of Study XV explained this value was due to the low resistance that particles of such size span imposed on the flow of the silica fume-modified cement paste medium used in that work [17]. Note that this study was not included in Figure 14 since it made the majority of the data too congested around the $(0,0)$ corner of the plots. A full list of the references reported in this table, under the column "reference study", can be found in References B. This review of the literature suspension rheology revealed that most of the studies cited performed tests with maximum particle sizes less than $0.5 \mathrm{~mm}(500 \mu \mathrm{m})$. No reference was found using $1 \mathrm{~mm}$ beads in a non-Newtonian medium. Interestingly, the relative viscosities reported varied from 1.3 to 22 . This wide range could be attributed to the spindle used, the shear rate used, and the suspended particle concentration. Thus, this literature review clearly showed inconsistencies in the measured viscosities. Experimentally determined viscosities were generally lower when compared to theoretical values. 
Table 6: Relative viscosities from the literature for mixtures similar to our $\mathbf{4 0} \%$ SRM 2493 mortar. The various particle sizes analyzed in those studies are also displayed. The $1000 \mathrm{\mu m}$ data point from Study XV was not included in Figure 14 for $\mathrm{x}$-axis clarity.

\begin{tabular}{|ccc|}
\hline $\begin{array}{c}\text { Reference } \\
\text { Study }\end{array}$ & $\begin{array}{c}\text { Max Particle Size } \\
{[\mu \mathrm{m}]}\end{array}$ & $\begin{array}{c}\text { Relative } \\
\text { Viscosity }\end{array}$ \\
\hline I & 0.05 & 8.0 \\
II & 0.11 & 1.3 \\
III & 0.23 & 3.9 \\
IV & 0.64 & 6.0 \\
V & 0.89 & 5.0 \\
VI & 12 & 6.0 \\
VII & 30 & 5.9 \\
VIII & 40 & 6.0 \\
IX & 42.3 & 22.1 \\
X & 43 & 9.5 \\
XI & 50 & 3.0 \\
XII & 95 & 9.2 \\
XIII & 100 & 3.0 \\
XIV & 124 & 3.0 \\
XV & $200 *$ & 3.7 \\
XVI & $270 *$ & 6.5 \\
XV & 350 & 2.1 \\
XV & 1000 & 1.6 \\
\hline
\end{tabular}

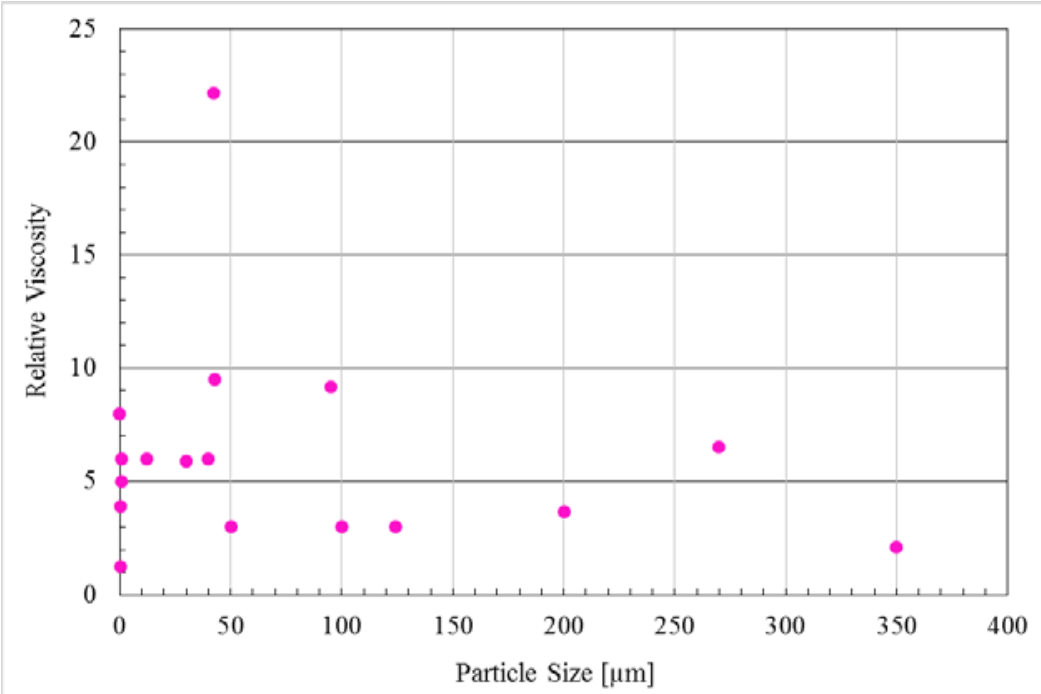

Figure 14: Various particle sizes reported in the literature yielded various relative viscosities for suspensions similar to this study. 


\section{Summary}

The data obtained from tests in this study originally were used for the purpose of obtaining certification for SRM 2493. Certification testing typically requires a large set of data with enough data points to be statistically valid. During the analysis of the certification data, two findings occurred, but only one of them was expected: 1) The experiments yielded reproducible data, and 2) tended to indicate a lower relative viscosity than the simulation model due to particle migration and slippage of the material when sheared.

\subsection{Recommended Tools}

This study showed that either an enclosed or open system consistently produced a decreasing accuracy trend in measured viscosity with the spiral the most accurate followed by the vane, with the cylinder being the least accurate. The spiral returned the highest viscosity readings, closest to the theoretical values. Thus, from the analysis completed during this study it is recommended that when testing materials similar to mortar, it is advantageous to use a spiral-like spindle. The use of such spindles allows mixing to keep occurring while testing ${ }^{4}$, which helps prevent or reduce sedimentation and radial migration of the particles. The spiral also eliminates a hard wall rotating in the center, eliminating a difference in packing density of the particles near a surface, thus reducing slippage.

\subsection{Influence of the spiral manufacturing}

The spirals used at NIST were produced by 3D printing with acrylonitrile butadiene styrene (ABS) and polylactic acid (PLA). Then, a metal axis was placed in the center to connect with the rheometer. The whole production can take less than a day and is inexpensive to manufacture as 3D printers become more widely available. Early tests showed that a plastic shaft was not rigid enough to ensure proper torque measurements and that is the reason that the 3D printed spiral had a metal shaft. NIST will post on the SRM 2493 website the file containing the information necessary to print the spiral. Thus, the question was how to ensure that different labs could produce

\footnotetext{
${ }^{4}$ Didier Lootens (SIKA) provided NIST with the initial design of the spiral
} 
similar data when using spirals printed with different plastic and devices. A small study was conducted by testing six different spirals on the same SRM 2492 and SRM 2493 material.

Table 7 describes the spiral tools that have been produced and investigated, along with which printer and type of material was used. Three printers are represented, one with dual extruders and the others with a single extruder for support and model material. Additionally, two of the most common 3D plastic printing materials, ABS and PLA, were used. The errors and averages presented in the data include the assumption of different materials, printers, timing, and minimal alterations to the dimensions.

Most of the data presented earlier in this paper was obtained with the RHN-83C spindle, which unfortunately broke before it could be used for this comparative test. Observation of the spirals shows various evident structural imperfections due to a lack of manufacturing precision. A digital caliper was used to measure the diameters and lengths of each tool produced. Figure 15 displays a schematic of how the tools were measured. The tools are pictured in Figure 16. 
Table 7: Summary of double spiral tools varying in manufacturing characteristics. The nominal tool diameter was $\mathbf{3 5 . 0 0} \mathbf{~ m m}$ and the length was $\mathbf{5 0 . 0 0} \mathbf{~ m m}$. The uncertainty of the caliper was $0.05 \mathrm{~mm}$

\begin{tabular}{|l|l|l|c|c|c|}
\hline NIST Code & \multicolumn{1}{|c|}{$\begin{array}{c}\text { Date } \\
\text { Manufactured }\end{array}$} & \multicolumn{1}{|c|}{ 3D Printer } & Material & $\begin{array}{c}\text { Diameter } \\
\text { (see Figure 15) } \\
(\mathrm{mm})\end{array}$ & $\begin{array}{c}\text { Length } \\
(\mathrm{mm})\end{array}$ \\
\hline RHN-83A & 15 October 2014 & Dual Extruder & ABS & 35.0 & 50.0 \\
\hline RHN-108A & 10 September 2015 & Dual Extruder & ABS & 34.3 & 48.6 \\
\hline RHN-108B & 11 September 2015 & Dual Extruder & ABS & 34.7 & 49.1 \\
\hline RHN-133A & 11 December 2015 & Single Extruder 1 & PLA & 35.0 & 49.6 \\
\hline RHN-133B & 11 December 2015 & Single Extruder 1 & PLA & 35.0 & 49.5 \\
\hline RHN-133C & 16 December 2015 & Single Extruder 2 & PLA & 35.1 & 48.5 \\
\hline RHN-133D & 31 December 2015 & Single Extruder 1 & ABS & 34.9 & 49.5 \\
\hline \multicolumn{7}{r|}{ Average [mm] } & $\mathbf{3 4 . 9}$ & $\mathbf{4 9 . 2}$ \\
\hline \multicolumn{7}{|r|}{ Standard Deviation [mm] } & 0.3 & 0.6 \\
\hline \multicolumn{7}{|r}{ COV [\%] } & $0.8 \%$ & $1.2 \%$ \\
\hline
\end{tabular}

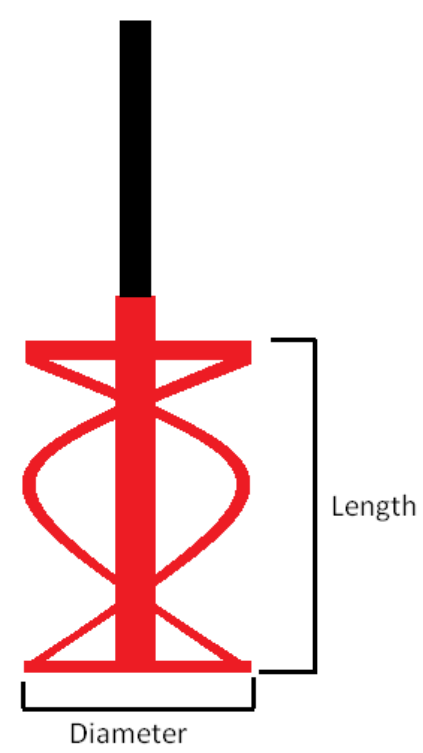

Figure 15: Schematic of Spiral Dimensions 


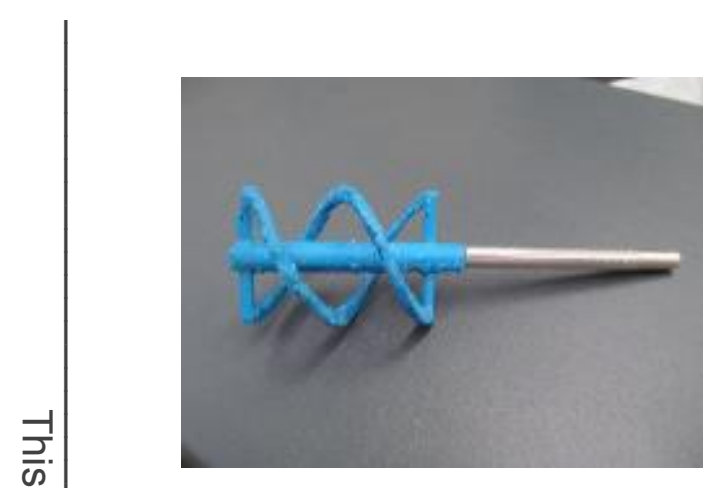

A: $R H N-108 A$

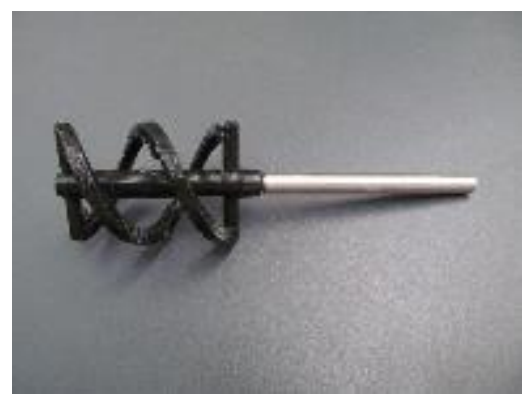

D: $R H N-133 B$

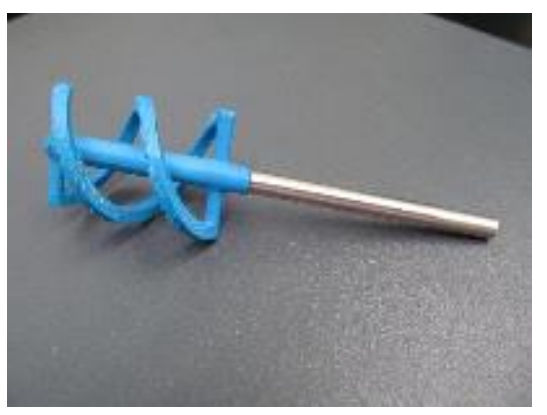

B: $R H N-108 B$

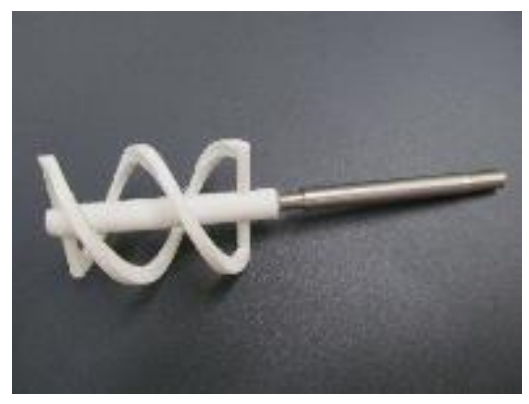

E: $R H N-133 C$

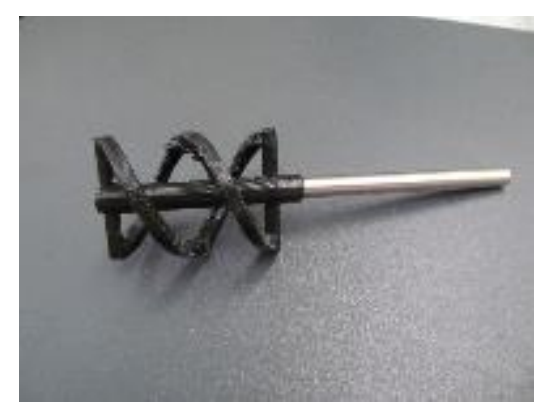

C: $R H N-133 A$

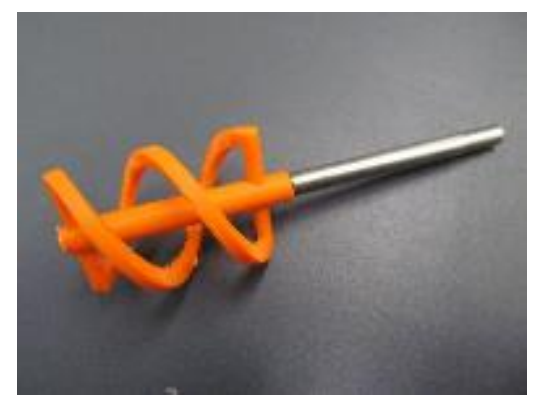

F: $R H N-133 D$

Figure 16: Double Spiral Tools Utilized. See Table 5 for details on the spirals

The rheometer parameters, which were used for all tests utilized in this study, were: the rheometer temperature was set to $23{ }^{\circ} \mathrm{C} \pm 1{ }^{\circ} \mathrm{C}$ before shearing the material with rotational steps ranging from $0.1 \mathrm{~min}^{-1}$ to $100 \mathrm{~min}^{-1}$, and back down to $0.1 \mathrm{~min}^{-1}$. All tests performed with a helical spindle incorporated a gap of $15 \mathrm{~mm}$ between the end of the tool and the bottom of the serrated cup (Table 7).

Three different mixtures nominally similar to SRM 2492 and SRM 2493 were generated for the purpose of the investigation. All three mixtures differed from the SRMs as they were produced using the same limestone and corn syrup, but not from the actual packaged SRM certified boxes. Therefore, the spiral tool comparison was conducted using a paste similar to SRM 2492 (no glass beads $-0 \%$ ) and mortar similar to SRM 2493 (25\%, and $40 \%$ by volume of $1 \mathrm{~mm}$ glass beads), but the paste and mortar were not exactly the same as the SRMs. Hence, the results are not expected to reflect the data obtained in the certificate, especially as the corn syrup was from a different lot number. Nevertheless, the mixtures were produced using the same procedure as described for the SRMs. 
The raw data for the paste are presented in Figure 17. The data are presented as the torque and rotational speed without a transformation for the calibration to ensure that any discrepancy between the spirals could be clearly highlighted. Figure 17 shows the average data obtained for five spirals (RHN-83A was broken already) measured three times, stirring the material between each test. It should be noted that the largest standard deviation over the three trials was for batch 1 at $11 \%$, while batch 2 and batch 3 were $7 \%$ and $9 \%$, respectively. Issues with sealing the blender were encountered at the time of mixing batch 1 , resulting in a potential loss of water during mixing, which may have contributed to such a large standard deviation. In the SRM 2492 certificate, the relative uncertainty was $9 \%$ [13]. Thus, it is clear that batch 2 and batch 3 are within one standard deviation of the data collected using one spiral and a larger number of tests, and batch 1 is within reasonable uncertainty.

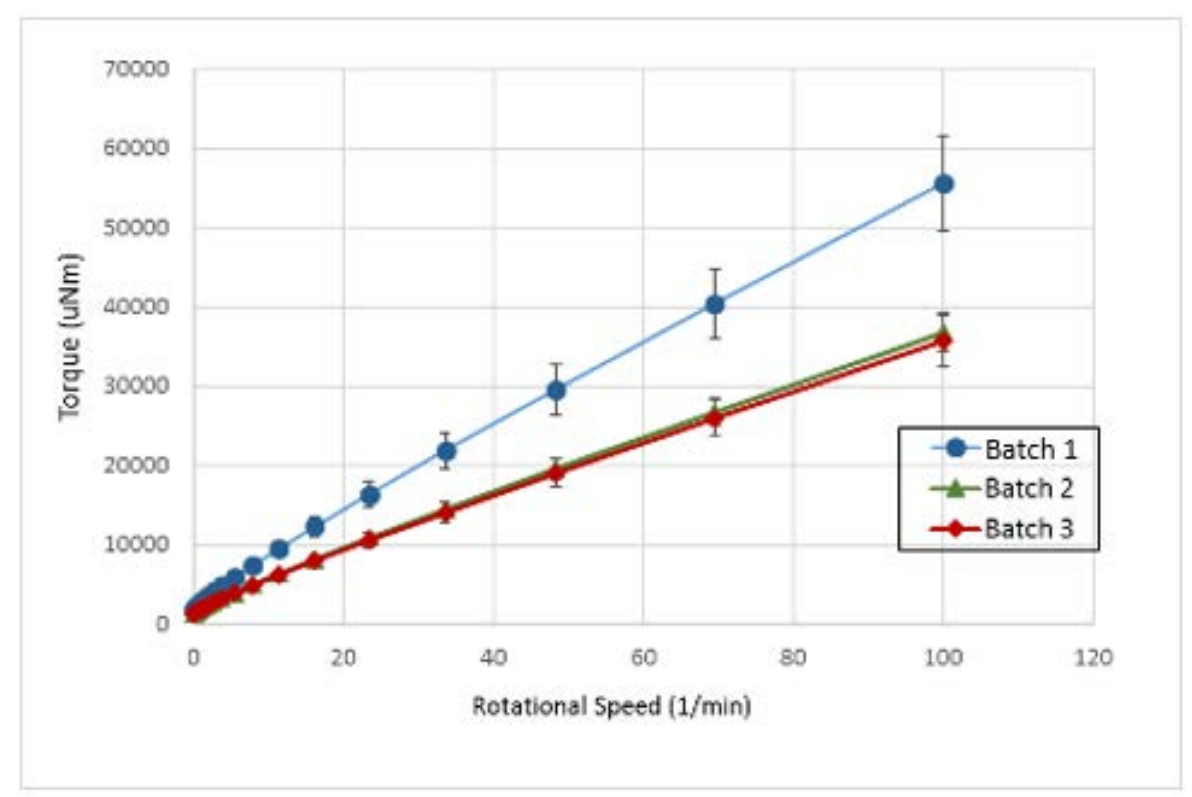

Figure 17: Paste tests - average results. Uncertainty represents one standard deviation of all measurements with 5 different spirals.

Using two of the paste batches (Batch 2 and Batch 3) described previously, $1 \mathrm{~mm}$ glass beads were added to the mixtures to create a mortar with aggregate concentrations of $25 \%$ and 40 \% by volume, respectively. Figure 18 displays the results obtained. For bead concentrations at 25 $\%$ by volume, the maximum relative uncertainty is $9 \%$, while for the bead concentrations at 40 $\%$, the maximum relative uncertainty is about $13 \%$. The relative error of one spiral in the 
development of SRM 2493 [4] was $15 \%$. It is likely that the uncertainty could be reduced if the dimensions of the spiral were better controlled during the 3D printing process. For instance, it was noted that spirals with both a larger radius and longer length tend to produce higher torque readings. Dimensional precision may be found through more accurate printing devices or using plastic with lower coefficients of thermal expansion.

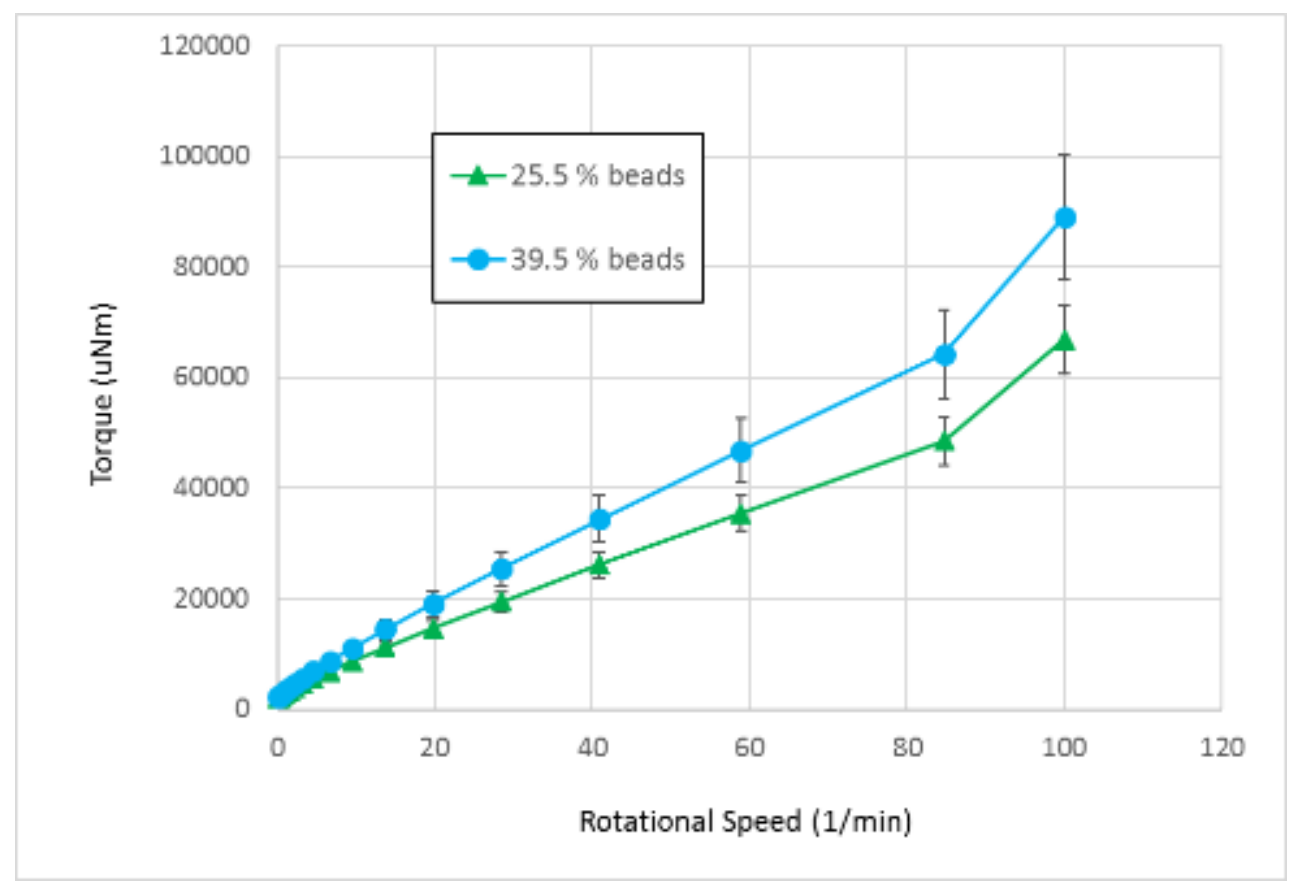

Figure 18: Mortar results averages. Uncertainty represents one standard deviation of all measurements with 5 different spirals

In conclusion, the uncertainty introduced by the variability in the 3D manufacturing of the spiral spindle is smaller or comparable to the uncertainty of the certified values of the SRM suspension. However, as the usage of SRM 2493 requires obtaining a base line with the paste before introducing the beads, any discrepancy in measurement due to manufacturing inconsistency should not impact the results. In other words, the ratio between the paste and the mortar is insensitive to the spiral used. 


\section{Conclusion}

This study has identified issues that affect the accuracy of the rheological measurements obtained from testing SRM 2493 with a rotational rheometer. The goal was to gain fundamental insight about the impact of various spindle families (cylindrical, vane and spiral) on the shear measurements of suspensions through comparison of experimental testing and computer simulation of an ideal Couette flow. It was found that rheological results were dependent on the spindle used. Specifically, a decreasing trend in accuracy of experimental flow curves with respect to computer simulated flow curves was highlighted, with the lowest accuracy (i.e., highest deviation between model and experimental results) being obtained with the cylindrical spindle and the best agreement obtained with the spiral spindle. The difference is attributed to increased slippage for the cylindrical spindle compared to a spiral spindle. Also particle migration away from the shearing surface is reduced with a spiral spindle, due to its mixing action during the measurements.

In this study, experimental results for the $20 \%$ volume concentration mortars matched the prediction model reasonably well. On the other hand, mortars with $40 \%$ volume concentration exhibit lower viscosity than the simulation values based on a pure couette geometry. The discrepancy between the theory and the experimental increases with the volume fraction and could be attributed to the presence of a free surface and other boundary conditions not considered in the simulation.

Finally, an extensive literature review was conducted, aimed at finding a correlation between particle size and relative viscosity when testing a mortar composed of particles concentrated at about $40 \%$ by volume. It was found that the relative viscosity values reported ranged over an order of magnitude, probably depending on the rheometer geometry and experimental conditions. A surprising finding from the review is that no studies were conducted using $1 \mathrm{~mm}$ particles in a nonNewtonian matrix.

In summary, it is recommended to use a spiral spindle, easily built with a 3D printer, for measurements with materials like mortar containing large particles. Also, the rheometer should be calibrated using a non-Newtonian paste like material, e.g. SRM 2493, and then tested with the same material containing beads. 


\section{References}

[1] C. F. Ferraris and L. E. Brower, "Comparison of concrete rheometers: International tests at LCPC (Nantes, France) in October 2000 (NISTIR 6819)," National Institute of Standards and Technology, Gaithersburg, MD.

[2] C. F. Ferraris and L. E. Brower, "Comparison of concrete rheometers: International tests at MB (Cleveland OH, USA) in May 2003 (NISTIR 7154)," National Institute of Standards and Technology, Gaithersburg, MD.

[3] A. Olivas, C. F. Ferraris, W. F. Guthrie and B. Toman , "Re-certification of SRM 2492: Bingham Paste Mixture for Rheological Measurements," National Institute of Standards and Technology. (NIST Special Publication 260-182). U.S. Department of Commerce, Gaithersburg, MD, August 2015.

[4] A. Olivas, C. F. Ferraris, N. S. Martys, E. J. Garboczi and B. Toman, "Certification of SRM 2493: Standard Reference Mortar for Rheological Measurements," National Institute of Standards and Technology (2015). NIST Special Publication 260-xxx. U.S. Department of Commerce., Gaithersburg, MD, 2015.

[5] R. Ferron, "Introduction to Rheology," The University of Texas at Austin, Austin, Texas, 2015.

[6] P. J. Cullen, "Rotational rheometry using complex geometries - a review.," Texture Studies, vol. 34, p. 120, 2003.

[7] H. Hafid, G. Ovarlez, F. Toussaint, P. H. Jezequel and N. Roussel, "Assessment of Potential Concrete and Mortar Rheometry Artifacts using Magnetic Resonance Imaging," Cement and Concrete Research, vol. 71, pp. 29-35, 2015.

[8] A. I. Jomha, M. F. Edwards and L. V. Woodcock, "New method for predicting the power requirement for mixing shear thinning suspensions.," Chemical Engineers Science, vol. 45, pp. 1389-1396, 1990.

[9] M. N. F. C. D. K. D. Shu H., "A numerical study for the flow of Bingham-like Fluids in two Dimensional Vane and Coaxial cylinders using Smoothed Particle Hydrodynamic (SPH) based Method"," J. of Non-Newtonian Fluid Mechanics, vol. 165, pp. 363-375, 2010.

[10] D. Hugelshofer, E. J. Windhab and J. Wang, "Rheologoical and structural changes during the mixing of suspensions and emulsions.," Applied Rheology, vol. 10, pp. 22-30, 2000. 
[11] L. Choplin and P. Marchal, "Systematic rheology or how to extend the progress viscosity concept.," in Proceedings of XIIth International Congress on Rheology, Quebec, QC, Canada, 1996.

[12] ASTM, "C1738/C1738-14: Standard Practice for High-Shear Mixing of Hydraulic Cement Pastes," ASTM International, West Conshohocken, PA, 2015.

[13] A. Olivas, C. F. Ferraris , E. J. Garboczi and W. F. Guthrie, "Re-certification of SRM 2492: Bingham Paste Mixture for Rheological Measurements," National Institute of Standards and Technology. (NIST Special Publication 260-xxx). U.S. Department of Commerce, Gaithersburg, MD, 2012.

[14] L. Maxime, N. S. Martys, W. L. George, D. Lootens and P. Hebraud, "Scaling laws for the flow of generalized Newtonian suspensions," Journal of Rheology , vol. 58, 2014.

[15] M. Allen and D. Tildesley, Computer simulations of liquids, Clarendon, Oxford, 1987.

[16] N. S. Martys, W. L. George, B.-W. Chun and D. Lootens, "A smoothed particle hydrodynamics-based fluid model with a spatially dependent viscosity: application to flow of a suspension with a non-Newtonian fluid matrix.," Rheologica acta, vol. 49, no. 10, pp. 1059-1069, 2010.

[17] L. P. Esteves , P. B. Cachim and V. M. Ferreira , "Effect of fine aggregate on the rheology preoperties of high performance cement-silica systems," Construction and Building Materials, vol. 24, pp. 640-649, 2010.

[18] M. F. Edwards, A. I. Jomha, W. C. MacSporran and L. V. Woodcook, "The power requirement for mixing concentrated solid/liquid suspensions.," in Institute of Chemical Engineers Annual Research Meeting, April 14-15, Bradford, U.K. , 1986.

[19] M. F. Edwards and A. I. Jomha, "Dispersions and emulsions.," in Institute of Chemical Engineers, Delft, Netherlands, 1987.

[20] F. C. Hackley V.A., "The Use of Nomenclature in Dispersion Science and Technology," NIST, 2001. 


\section{References B}

This list was included to clarify part of the literature review section 4.4, specifically referring to Table 4. In that table is a list of studies reviewed, which are found under the column "reference study”. The full citations for these are included in this list of references.

[I] D. A. R. Jones, B. Leary, Boger D. V., "The rheology of a concentrated colloidal suspension of hard spheres," Colloidal and Interface Science, vol. 147 (2), 479-495, 1991.

[II] J.C. van der Werff, C. G. de Kruif, "Hardsphere Colloidal Dispersions: The scaling of rheological properties with particle size, volume fraction, and shear rate," Journal of Rheology, vol. 33, 421-454, 1989.

[III] T. Shikata, D. S. Pearson, "Viscoelastic behavior of concentrated spherical suspensions," Journal of Rheology, vol. 38, 601-616, 1994.

[IV] S. Phan, W. B. Russel, Z. Cheng, J. Zhu, P. Chaikin, J. H. Dunsmuir, R. H. Ottewill, "Phase transition, equation of state, and limiting shear viscosities of hard sphere suspensions," American Physical Society, vol. 54 (6), 6633-6645, 1996.

[V] P. D'Haene, J. Mewis, G. G. Fuller, "Scattering Dichroism Measurements of flow-induced structure of a shear thickening suspension," Colloidal and Interface Science, vol. 156, 350-358, 1993.

[VI] B. K. Aral and D. M. Kaylon, "Viscoelastic material functions of noncolloidal suspensions with spherical particles," Journal of Rheology, vol. 41, 599-620, 1997.

[VII] L. Nicodemo and L. Nicolais., "Viscosity of bead suspensions in polymeric solutions," Applied Polymer Science, vol. 18, 2809-2818, 1974. 
[VIII] S.C. Tsai, D. Botts and J. Plouff, "Effects of particle properties on the rheology of concentrated noncolloidal suspensions," Journal of Rheology, vol. 36, 1291-1304, 1992.

[IX] S-C. Dai, F. Qi, R. I. Tanner, "Viscometric functions of concentrated non-colloidal suspensions of spheres in a viscoelastic matrix," Journal of Rheology, vol. 58, 183-198, 2014.

[X] I. E. Zarraga, D. A. Hill, D. T. Leighton Jr., "Normal Stresses and free surface deformation in concentrated suspensions of noncollodial spheres in a viscoelastic fluid.," Journal of Rheology, vol. 45, 1065-1084, 2001.

[XI] J.S. Chong, E.B. Christiansen, and A.D. Baer, "Rheology of concentrated suspensions," Applied Polymer Science, vol. 15, 2007-2021, 1971.

[XII] T.B. Lewis and L.E. Nielsen, "Viscosity of dispersed and aggregated suspensions of spheres," Journal of Rheology, vol. 12, 421-422, 1968.

[XIII] C. Chang and R.L. Powell, "Effect of particle size distribution on the rheology of concentrated bimodal suspensions," Journal of Rheology, vol. 38, 85-98, 1994.

[XIV] K. H. Sweeny and R. D. Geckler, "The Rheology of Suspensions," Applied Physics, vol. 25, 1135-1144, 1954.

[XV] L. P. Esteves, P.B. Cachim, and V.M. Fereira, "Effect of fine aggregate on the rheology properties of high performance cement-silica systems" Construction and Building Materials, vol. 24, 640-649, 2010.

[XVI] C. Moreland, "Viscosity of suspensions of coal in mineral oil," Canadian Journal of Chemical Engineering, 24-28, 1963. 